\title{
Numerical simulation of the formation of a spiral galaxy
}

\author{
P. R. Williams ${ }^{1,2}$ and A. H. Nelson ${ }^{3}$ \\ 1 Department of Physics and Astronomy, Cardiff University, PO Box 913, Cardiff, CF2 3YB, UK \\ 2 Astronomical Institute, Tohoku University, Aoba, Sendai, 980-8578, Japan \\ 3 Department of Physics and Astronomy, Cardiff University, PO Box 913, Cardiff, CF2 3YB, UK \\ e-mail: nelsona@cardiff.ac.uk
}

Received 12 November 1999 / Accepted 2 May 2001

\begin{abstract}
A simulation is described in which the numerical galaxy formed compares favourably in every measurable respect with contemporary bright spiral galaxies, including the formation of a distinct stellar bulge and large scale spiral arm shocks in the gas component. This is achieved in spite of the fact that only idealized proto-galactic initial conditions were used, and only simple phenomenological prescriptions for the physics of the interstellar medium (ISM) and star formation were implemented. In light of the emphasis in recent literature on the importance of the link between galaxy formation and models of the universe on cosmological scales, on the details of the physics of the ISM and star formation, and on apparent problems therein, the implications of this result are discussed.
\end{abstract}

Key words. galaxies: spiral, formation, evolution, kinematics and dynamics, fundamental parameters methods: $N$-body simulations

\section{Introduction}

During the past decade our observational knowledge of the evolution of galaxies to high redshift has considerably improved (Bunker \& van Breugel 1999). On larger scales recent observations of the cosmic microwave background have given a greater confidence in the validity of the standard cosmological models (de Bernardis et al. 2000). However extensions of these cosmological models to galactic scales suggest that there are possibly serious discrepancies between the characteristics of galaxies predicted by galaxy formation in such cosmological contexts and those observed. Specifically, these theories predict too many dwarf galaxies in comparison to the numbers of satellite galaxies in the Local Group (Klypin et al. 1999; Moore et al. 1999a); the largest of disk galaxies are predicted to have too little angular momentum and thus are more centrally condensed than those observed (Navarro \& Benz 1991; Navarro \& White 1993; Navarro et al. 1995); and the predicted inner mass profiles of halos appear to be far steeper than those inferred from the rotation curves of dwarf and low surface brightness galaxies (Flores \& Primack 1994; Burkert 1995; de Blok \& McGaugh 1997; Hernandez \& Gilmore 1998; Moore et al. 1999b). Recent studies have highlighted potentially very important numerical issues related to these problems, particularly concerning the implementation of interstellar processes which occur on length scales much smaller than those presently resolvable in simulations;

Send offprint requests to: P. R. Williams, e-mail: williams@astr.tohoku.ac.jp and how such galacto-microscopic physics may be consistently and effectively implemented in such galaxy formation simulations (Yepes et al. 1997; Sommer-Larsen et al. 1999; Hultman \& Pharasyn 1999; Springel 2000). In addition, many possible physical and astrophysical solutions have also been suggested, for example, modifications to the dark matter physics (Sommer-Larsen \& Dolgov 1999; Spergel \& Steinhardt 2000; White \& Croft 2000; Firmani et al. 2000; Yoshida et al. 2000; Burkert 2000; Hannestad \& Scherrer 2000; Riotto \& Tkachev 2000); modifications to the manner in which the primordial power spectrum evolves (Kaminokowski \& Liddle 2000); and work emphasizing the importance of supernovae feedback and other interstellar physics to the gross structure of galaxies (Navarro et al. 1996; Navarro \& Steinmetz 1997; Weil et al. 1998; Gelato \& Sommer-Larsen 1999; Bullock et al. 2000).

In order to assess the importance of these recent developments, it is useful to view the current state of galaxy formation theory in the broader context of the development of galaxy formation theory to date. Gravitational collapse of dense proto-galactic clouds is now the standard paradigm for galaxy formation, and constituted the basis of the early galaxy formation scenarios of von Weizsäcker (1951) and Hoyle (1953). Using data on dwarf stars, Eggen et al. (1962) showed that the present structure of the Galaxy is consistent with such a collapse hypothesis, in that the oldest stars generally have high angular momentum. The observed rotation curves of disk galaxies are also in keeping with the collapse hypothesis, in that if protogalaxies initially gain their angular momentum through 
tidal torques, and angular momentum transport during collapse is not significant, galaxies have typically collapsed by a factor of 5-10 (Mestel 1963; Fall \& Efstathiou 1980). Also, exponential surface density profiles, a defining characteristic of observed stellar disks, are a natural consequence of such a scenario (Gunn 1982). Lynden-Bell (1967) demonstrated that if such a proto-galactic collapse is predominantly dissipationless, perhaps relevant to the formation of spheroidals, the proto-galaxy may reach equilibrium on a timescale much shorter than that expected from the star-star relaxation time, via violent relaxation.

To build on these early ideas, it was necessary to relax any restrictive assumptions imposed on the model physics or on the geometry of the evolving protogalaxy, and hence to use computer simulation. Early examples of such simulations can be found in, for example, van Albada (1982), Larson (1969, 1974, 1975, 1976), Gott \& Thuan (1976), Lake \& Carlberg (1988, 1988), Katz \& Gunn (1991), and Katz (1992), and the last two decades have seen a number of key developments which have allowed galaxy formation research to accelerate along this path. Firstly there has been a huge increase in the processing power of computers, and secondly efficient numerical techniques applicable to the galaxy formation problem have been developed, principally Treecode gravity (Barnes \& Hut 1986; Hernquist \& Katz 1989) and Smoothed Particle Hydrodynamics (Lucy 1977; Gingold \& Monaghan 1977; Hernquist \& Katz 1989; Monaghan 1992).

A proto-galaxy can be defined as a region of the universe that encloses the gas which will eventually constitute a galaxy just prior to this gas forming significant amounts of stars. Thus in order to construct a computer model of a protogalaxy and its evolution, one must specify:

1. the distribution of matter, in various forms, and kinetic energy at this early stage, and

2 . the physics governing the matter of which the protogalaxy is constituted.

The aim of computer simulation is then to find choices for (1) and (2) which are consistent with observations of galaxies in the process of forming and with the implications of cosmological models; and to eliminate the choices that result in numerical galaxies which are inconsistent with observations of contemporary galaxies.

The direction of recent simulation work has been to try to further our understanding of galaxy formation by coupling galaxy formation to cosmological models of the large scale structure of the universe (Navarro \& White 1993, 1994; Steinmetz \& Müller 1994, 1995; Navarro \& Steinmetz 1997; Weil et al. 1998); and by increasing the sophistication of the prescription of dissipational physics (Yepes et al. 1997; Navarro \& Steinmetz 1997; Weil et al. 1998; Hultman \& Pharasyn 1999; Springel 2000). While it is natural for galaxy formation to develop in such directions, it must be recognized that the observational constraints on these issues are not strong. While significant advances have been made in probing galaxies to higher and higher redshifts, and in characterizing the gas content of the Universe as a function of redshift, we are still without direct observational evidence of a galaxy formation event. The life--cycle of galaxies cannot yet be observed in a statistical manner, as has been done for stars. Thus observational data cannot yet directly impose strong constraints on points (1) or (2).

However there are a number of things that can be inferred from observations of contemporary galaxies. Firstly, galaxies are found to have characteristic length and mass scales (Rees \& Ostriker 1977), while there are no such scales associated with gravitation. Furthermore, the fact that galaxies have gaseous and stellar disks indicates that significant levels of star formation cannot have occurred prior to or during their collapse. Thus it is strongly suggested that dissipational physics played an important role in the formation of galaxies.

This in turn implies that a cosmological power spectrum of density fluctuations (based on gravity alone) which is able to successfully reproduce the large scale structure of the Universe, is not necessarily relevant to galaxy formation on smaller scales. Such a power spectrum, when extrapolated to sub-protogalactic scales, lacks the effects of gas physics, which may play a dominant role in determining the noise spectrum on these scales during the epoch of galaxy formation.

Thus, while the long term aim of galaxy formation theory must be to develop an integrated picture of galaxy formation in a cosmological context, it is at present still productive to investigate galaxy formation neglecting cosmological considerations. This will allow us to investigate the mechanisms of self-gravitating gaseous collapse which may be relevant to galaxy formation, and to ascertain to what extent comparison of the final result with contemporary galaxies can put constraints on (1) and (2). Following this line of reasoning, and given our present limited understanding of the interstellar medium, we investigate here the extent to which the simplest choices of initial conditions and physics for a protogalaxy, when evolved into the fully non-linear regime, can reproduce the properties of contemporary galaxies. It turns out that the such simulations reproduce these properties remarkably well.

\section{Method}

\subsection{Initial conditions and input physics}

The initial conditions were a uniform density sphere of $10 \%$ gas and $90 \%$ collisionless dark matter by mass, in solid body rotation, and with a radial velocity directly proportional to radius. The particles were initially laid down on a uniform grid. This matter configuration is defined by four parameters: the total mass $M$, the initial radius $R_{\mathrm{i}}$, the initial angular velocity $\Omega_{\mathrm{i}}$, and a radial velocity scaling constant $H_{\mathrm{i}}$. The values used in this simulation were $M=5 \times 10^{11} M_{\odot}, R_{\mathrm{i}}=175 \mathrm{kpc}, \Omega_{\mathrm{i}}=0.161 \mathrm{Gyr}^{-1}$, and $H_{\mathrm{i}}=560 \mathrm{~km} \mathrm{~s}^{-1} \mathrm{Mpc}^{-1}$. This is equivalent to a total angular momentum $J \simeq 6 \times 10^{67} \mathrm{~kg} \mathrm{~m}^{2} \mathrm{~s}^{-1}$, a total 
energy of $E \simeq-4.6 \times 10^{51} \mathrm{~J}$, and thus a spin parameter of $\lambda_{\mathrm{i}}=G J^{-1}|E|^{1 / 2} M^{5 / 2} \simeq 0.06$ (Peebles 1969).

For the purposes of modeling galaxy formation at the spatial resolution presently possible in galaxy formation simulations, a model of the ISM which describes the bulk behaviour of interstellar gas on scales of several tens of parsecs and larger is required. In order to construct such a macroscopic model it is reasonable to first consider the state of interstellar gas in the solar neighbourhood and in external spiral galaxies. The dominant gas component in the local ISM is found to be thermodynamically cold, less than $10^{2} \mathrm{~K}$; dense, greater than $10 \mathrm{~cm}^{-3}$; and magnetized to a dynamically significant level. It is this cloud phase which dominates the bulk motions of gas in galaxies (Binney \& Merrifield 1998). Both the velocity dispersion of small clouds in the Solar neighbourhood (Stark \& Brand 1989) and the velocity dispersion which characterizes the bulk motions of gas in spiral galaxies is found to be of order $10 \mathrm{~km} \mathrm{~s}^{-1}$ (Spitzer 1978). Thus a model of the ISM in which gas is in the form of an ensemble of small clouds which themselves behave as a collisional fluid was adopted. Analogous to an ideal gas, the gas of small clouds is characterized by a bulk velocity dispersion $c_{\mathrm{s}}$, and a mass density $\rho$, which together provide a source of pressure $P=c_{\mathrm{s}}^{2} \rho$. In this simulation $c_{\mathrm{s}}=7.5 \mathrm{~km} \mathrm{~s}^{-1}$ was used, based on the measured velocity dispersion of clouds in the local ISM (Stark \& Brand 1989). Such a simple model for the ISM was first suggested by Cowie (1980), and this model may be considered as a first order approximation to a detailed description of the multi-phase ISM (McKee \& Ostriker 1977). The Cowie model has in effect been used extensively in simulations of galaxy dynamics and galaxy mergers (Theis \& Hensler 1993; Mihos \& Hernquist 1994; Englmaier \& Gerhard 1997).

The star formation rate density $\dot{\rho}_{*}$ was determined by a Schmidt law (Schmidt 1959) $\dot{\rho}_{*}=k_{\mathrm{s}} \rho_{\mathrm{g}}^{n_{\mathrm{s}}}$, where $\rho_{\mathrm{g}}$ is the total gas density. From observations of the global star formation rate density in spiral galaxies, a Schmidt law index $n_{\mathrm{s}} \simeq 1.5$ has been deduced and was used here (Buat 1992; Kennicutt 1997). A wide range of simple theoretical models of star formation in spiral galaxies also produce a similar value (Wyse 1986; Larson 1988, 1992; Kennicutt 1989, 1997; Wyse \& Silk 1989; Elmegreen 1994; Sleath \& Alexander 1995). The constant $k_{\mathrm{s}}$ was chosen such that for a typical present day ISM gas density $\rho_{\mathrm{g}}=0.042 M_{\odot} \mathrm{pc}^{-3}$ (Binney \& Tremaine 1987), and for a total galaxian gas mass of $m_{\mathrm{g}}=5 \times 10^{9} M_{\odot}$ (Hodge 1994), the implied global star formation rate $\dot{m}_{*} \simeq$ $0.5 M_{\odot} \mathrm{yr}^{-1}$, comparable to the measured present day star formation rates in intermediate to late type spiral galaxies (Kennicutt 1989). Based on these assumptions, the value $k_{\mathrm{s}}=0.461 M_{\odot}^{-1 / 2} \mathrm{pc}^{3 / 2} \mathrm{Gyr}^{-1}$ was used.

\subsection{Numerical details}

The numerical code was based on the Treecode-SPH algorithm described by Hernquist \& Katz (1989), modified to run on a distributed parallel computer, and to include an algorithm to model star formation. Full details of all the numerical methods and algorithms used, including tests and validations, are to be found in Williams (1998).

The gravitational softening length $\epsilon$ was kept constant in space and time with a value $\epsilon=175 \mathrm{pc}$. Gravitational accelerations were evaluated to quadrupole order, using a cell opening angle of $\theta_{c}=0.8$. The modified cell tolerance criteria given by Barnes (1994) was used to avoid errors in the calculation of gravitational accelerations such as those reported by Salmon \& Warren (1994).

The kernel radius of each $\mathrm{SPH}$ particle $h_{\mathrm{i}}$ was allowed to vary such that at all times between 30 and 50 neighbours were contained within $2 h_{\mathrm{i}}$. Kernel radii were evolved as described by Hernquist \& Katz (1989). However, occasionally this approach did not predict a kernel radius containing the required number of neighbours. In these cases, $h_{\mathrm{i}}$ was iteratively updated and the tree data structure was re-examined until the required number of neighbours within $2 h_{\mathrm{i}}$ was achieved. This process was found to incur little additional computational cost. The artificial viscosity prescription of Gingold \& Monaghan (1983) was used, with viscosity parameters $(\alpha, \beta)=(1,2)$.

As the simulation evolved, each SPH particle converted its gas mass into stellar mass at a rate controlled by a Schmidt law, and kept track of the amount of mass in gas and stars. Globally, when a small amount $M_{*}$ of the total gas mass had been converted into stellar mass, this stellar mass was re-distributed amongst $n_{*}$ new collisionless star particles of mass $m_{*}=M_{*} / n_{*}$. In this simulation $M_{*}=$ $5 \times 10^{7} M_{\odot}$, i.e. one thousandth of the initial gas mass, and $n_{*}=64$. The positions of these $n_{*}$ new star particles were chosen such that the recent star formation history and spatial distribution of newly formed stellar mass was statistically represented. Numerical tests indicate that this algorithm gives consistent results over a range of values for $M_{*}$ and $n_{*}$ (Williams 1998).

A second order individual particle timestep integration scheme was used to improve efficiency, as described by Hernquist \& Katz (1989). Each particle was assigned an individual timestep which is a power of two division of the largest allowed timestep, and a second order timecentred leapfrog integration scheme was used. Collisionless particles were assigned a timestep using the criteria given by Katz (1991), while the timestep for SPH particles was calculated using the minimum of this timestep and the SPH Courant condition given by Hernquist \& Katz (1989). A Courant number of $\mathcal{C}=0.1$ was used for all particles.

A simple method of parallelization was implemented in which the most computationally expensive parts of the Treecode-SPH algorithm, the evaluation of gravitational accelerations, the searching for SPH neighbours, and the calculation of SPH summations, were parallelized. All processors held all particle data in memory. Each processor was assigned an equal fraction of each particle species for the duration of the simulation, and calculated their gravitational accelerations, found their SPH neighbours, and calculated their SPH summations when required. 
The numbers of gas and dark matter particles used were 33552 and 33401 respectively. Conversion of all gas mass to stellar mass would have resulted in the formation of 64000 star particles. Particle data was output every $1.553 \times 10^{7} \mathrm{yr}$, and the simulation was evolved for a total of $8.570 \times 10^{9}$ yr. Typically 13 timestep levels were used, equivalent to a range in timesteps of 8192 . This increased to 32768 (15 timestep levels) during the most dynamically active periods of evolution, although, the distribution of particles amongst the timestep levels was found to have a long tail to small timesteps: at any given time, no more than $10 \%$ of particles had timesteps smaller than that of the 10th smallest timestep level. Thus the individual particle timestep integration scheme made significant savings in computational cost in comparison to a single timestep algorithm. The simulation took 15 days using 32 processors (roughly 1.3 CPU years) on the Cray T3E at the Edinburgh Parallel Computing Centre, as part of the Virgo Consortium's time allocation (Jenkins et al. 1998). This is equivalent to roughly $2 \times 10^{6}$ timestep iterations.

\section{Results}

Figures 1 to 6 show an evolutionary sequence of the gas, stellar, and dark matter. Each page refers to one instant in the simulation, and is labelled with the time from the beginning of the simulation $t_{\mathrm{sim}}$. All lengths are shown in kiloparsecs, although the range in spatial scale viewed generally varies from frame to frame. Particle plots are an effective way of illustrating the detailed structural features and the general evolution of a simulation such as that discussed here. However, when the dynamical range in density and the number of particles is large, such plots can be misleading to the eye in regions of extremum density. In low density regions the small number of particles can give the impression of empty holes, while these particles are really points in a tenuous continuum fluid. In high density regions particles can appear as a uniform solid mass, the actual density structure being completely saturated to the eye. Also, it is difficult to convey the very dynamical nature of simulations using only static images. With these limitations in mind, we encourage readers to supplement their reading of this paper by viewing the series of animations to be found at http:// www.astro.cf.ac.uk/pub/Alistair.Nelson/ indexgf.html.

Figures 7 and 8 show the final gas, stellar, and dark matter surface density profiles, and the final rotation curve of the numerical galaxy formed. The evolution of the global properties, and the structural parameters of the numerical galaxy versus time and redshift are shown in Figs. 9 to 13.

The formation and evolution of this numerical galaxy was found to fall naturally into five broad phases in which specific physical mechanisms and processes were found to dominate the evolution of the numerical galaxy. Each of the following sections is labelled with a rough estimate of the epoch to which the description applies, although it should be remembered that there are no distinct temporal boundaries between the phases.

\subsection{Phase I: Expansion (to $t_{\text {sim. }} \simeq 3 \mathrm{Gyr}$ )}

As the radial expansion was halted by gravity, the outer shell of mass reached maximum radius (roughly $300 \mathrm{kpc}$ ), see Fig. 1, although mass at smaller radii was still expanding. At this time, the dark matter particles still retained their initial grid structure to a high degree of accuracy, while the gas particles had to a greater extent dispersed from the initial lattice. This was due to the stochastic nature of errors in quantities evaluated using $\mathrm{SPH}$, and the propagation of these errors in time. Note that the errors in the individual particle positions have a negligible effect on the bulk distribution of matter. The low initial gas density resulted in a low initial star formation rate of $0.04 M_{\odot} \mathrm{yr}^{-1}$, which decreased smoothly to $0.02 M_{\odot} \mathrm{yr}^{-1}$ as the sphere of matter expanded and the gas density decreased. Only $7.5 \times 10^{7} M_{\odot}$ of gas was converted to stellar matter during this phase.

\subsection{Phase II: Collapse ( $t_{\mathrm{sim}} \simeq 3 \mathrm{Gyr}$ to $\left.t_{\text {sim. }} \simeq 6.2 \mathrm{Gyr}\right)$}

Successively inner shells of matter achieved zero radial velocity and began to fall in at an increasingly faster rate, see Figs. 2 and 3. As the collapse progressed, the mass distributions became flattened in the $x-z$ plane due to rotation. In the central regions of the swiftly deepening potential well, increasingly more dark matter collapsed past gas which was initially at the same radius. The highest densities encountered at the point of maximum collapse were of order $200 M_{\odot} \mathrm{pc}^{-3}$, although only roughly $6 \times 10^{9} M_{\odot}$ $(12 \%)$ of gas achieved such values.

By $t_{\text {sim. }} \simeq 6.2$ Gyr, Fig. 3, the gas distribution had begun to flatten to a disk-like structure, and viscously dissipate energy. As a consequence, layers of gas in the $x-y$ plane had begun to collide and shock in the equatorial plane. Because of inhomogeneity on scales of less than of order $1 \mathrm{kpc}$ in the distribution of gas particles on either side of the equatorial plane of the proto-galactic cloud, the gas was unable to viscously dissipate all kinetic energy in the direction perpendicular to the proto-galactic disk and shock to a perfectly thin disk. Thus, the gas clumps impacting on the plane of the proto-galactic disk exhibited damped oscillations about this plane. This is most clearly seen in the central regions of the numerical proto-galaxy, where the impact velocity onto the $x-y$ plane was largest, see Fig. 3. Although this behaviour was short lived, the high density gas maintained a sufficiently large amplitude of oscillation about the $x-y$ plane for a sufficient length of time to form stars in a roughly spherical distribution at the centre during the proceeding evolution, see Fig. 14 . Thus it is suggested that galactic bulges may form in this fashion. 

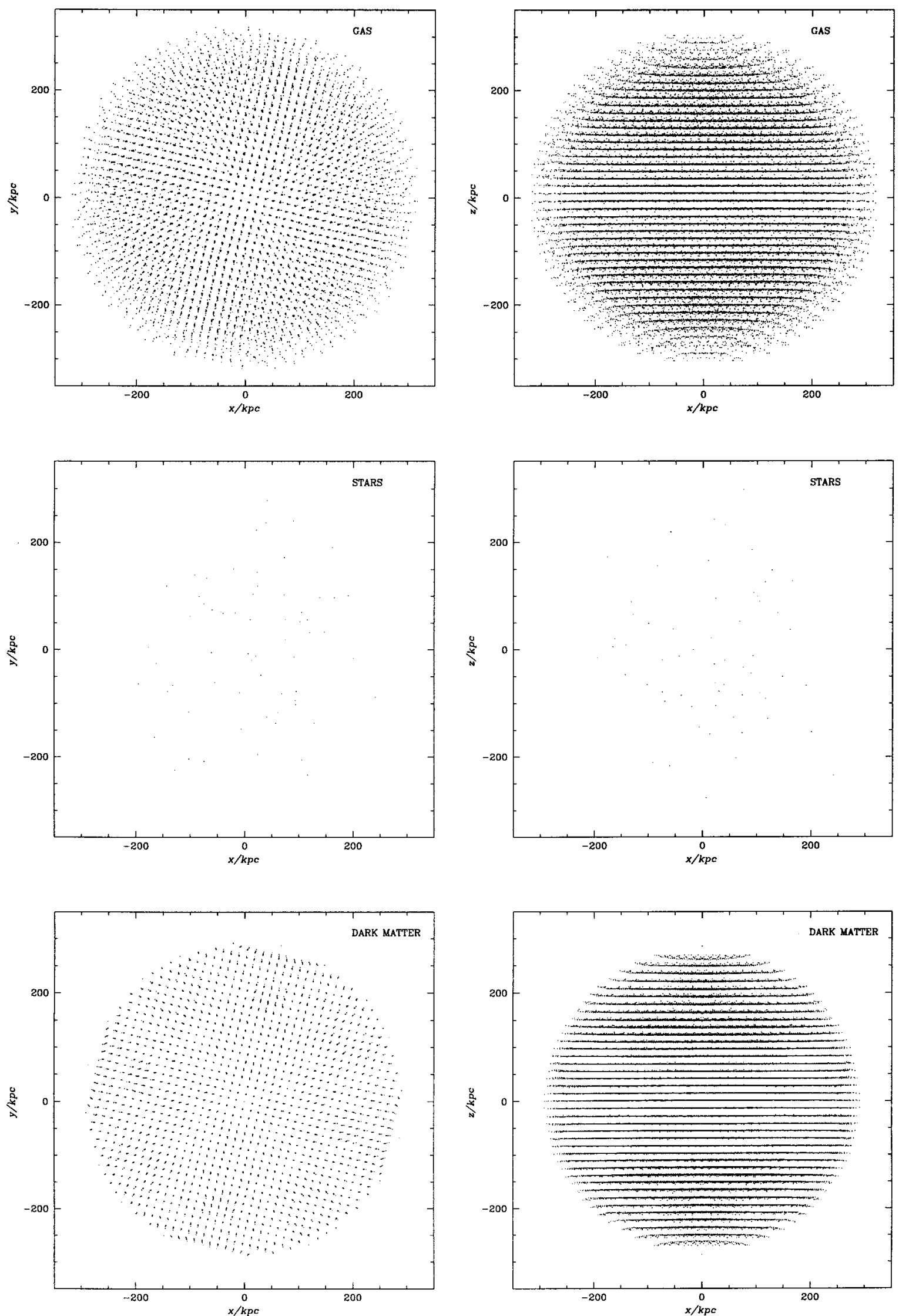

Fig. 1. $t_{\text {sim. }}=2.950$ Gyr. 



Fig. 2. $t_{\text {sim. }}=5.744$ Gyr. 

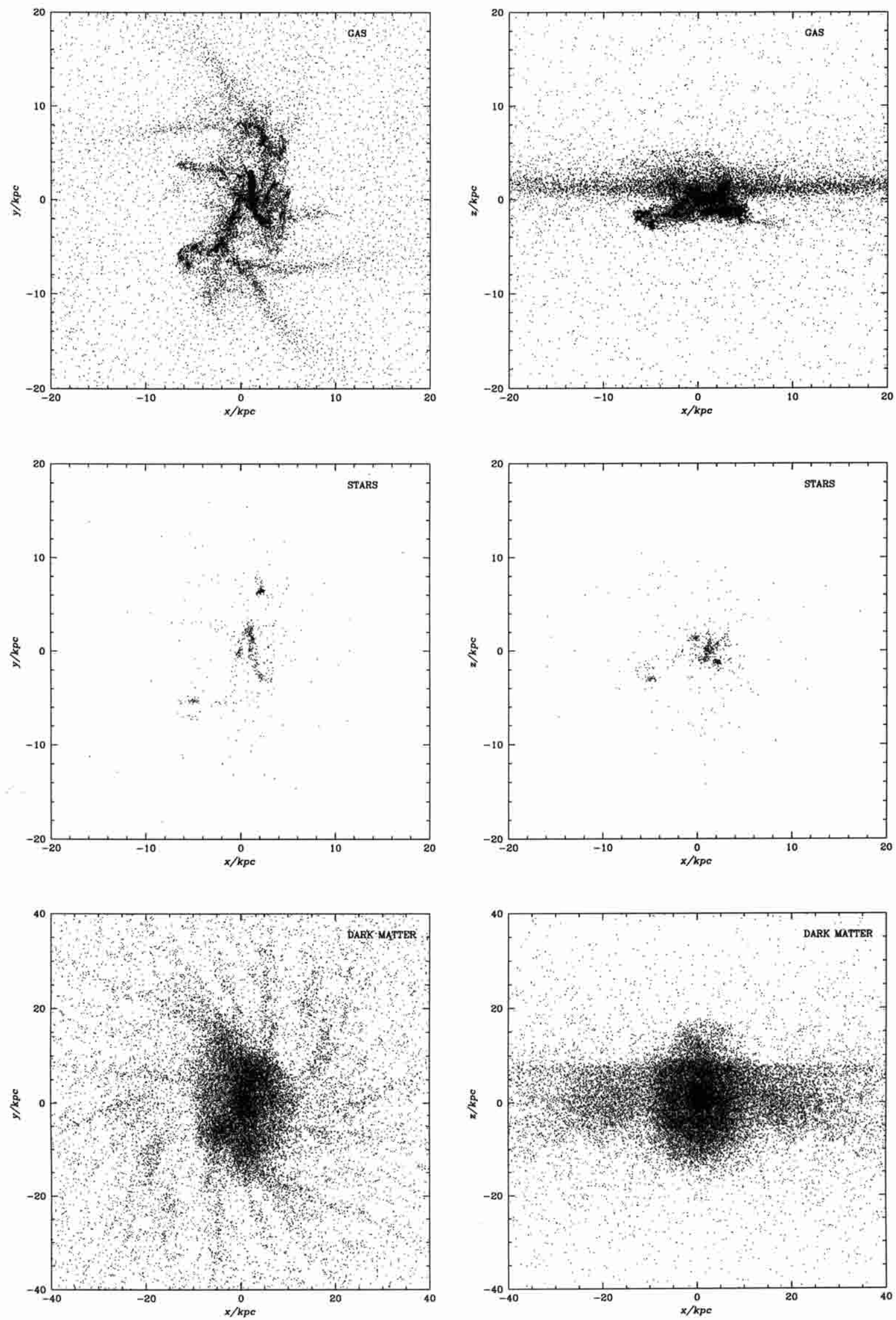

Fig. 3. $t_{\text {sim. }}=6.210$ Gyr. Note the decrease in scale from the previous figure. 

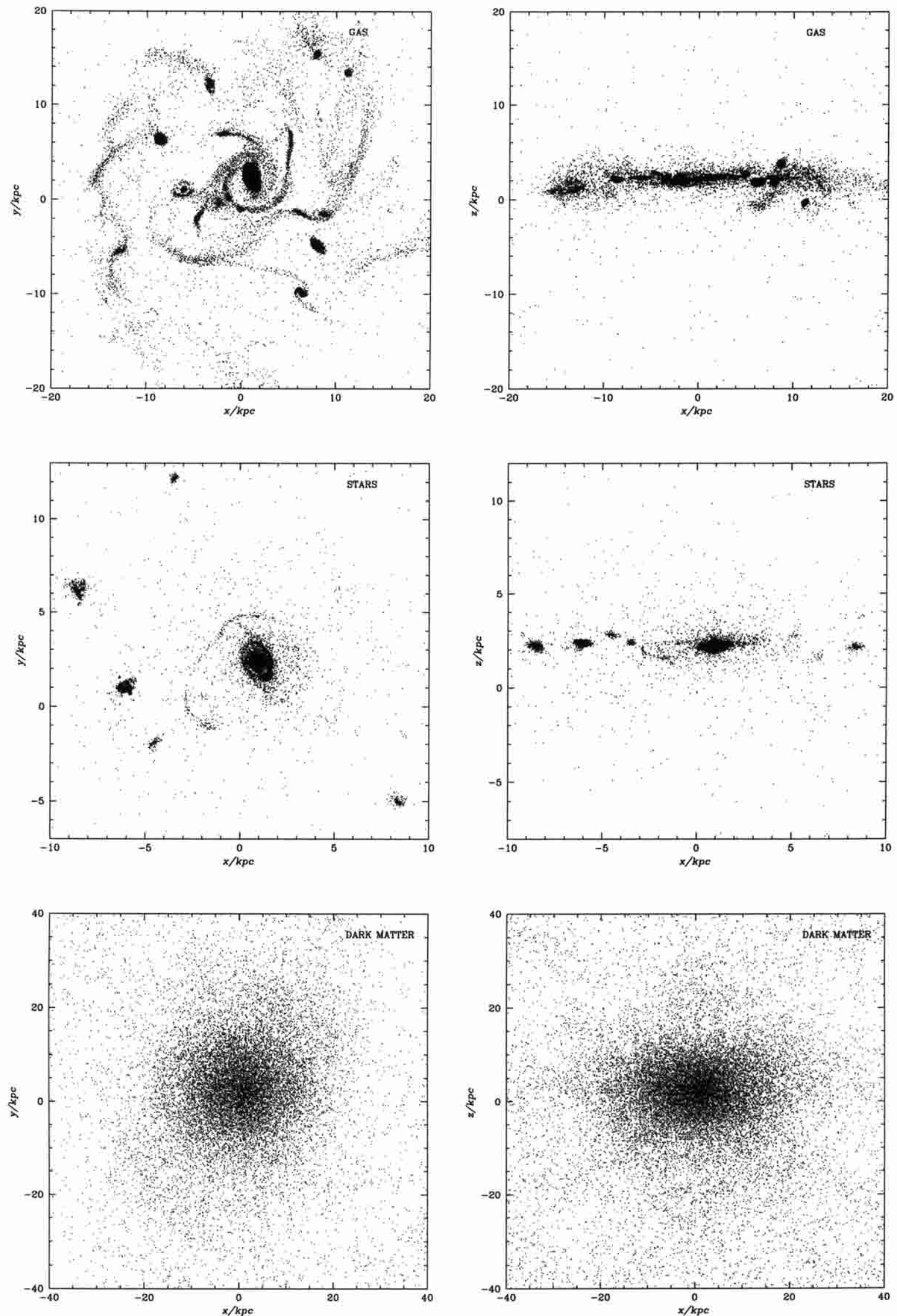

Fig. 4. $t_{\text {sim. }}=6.738$ Gyr. Note the reduced scale in the stellar images. 

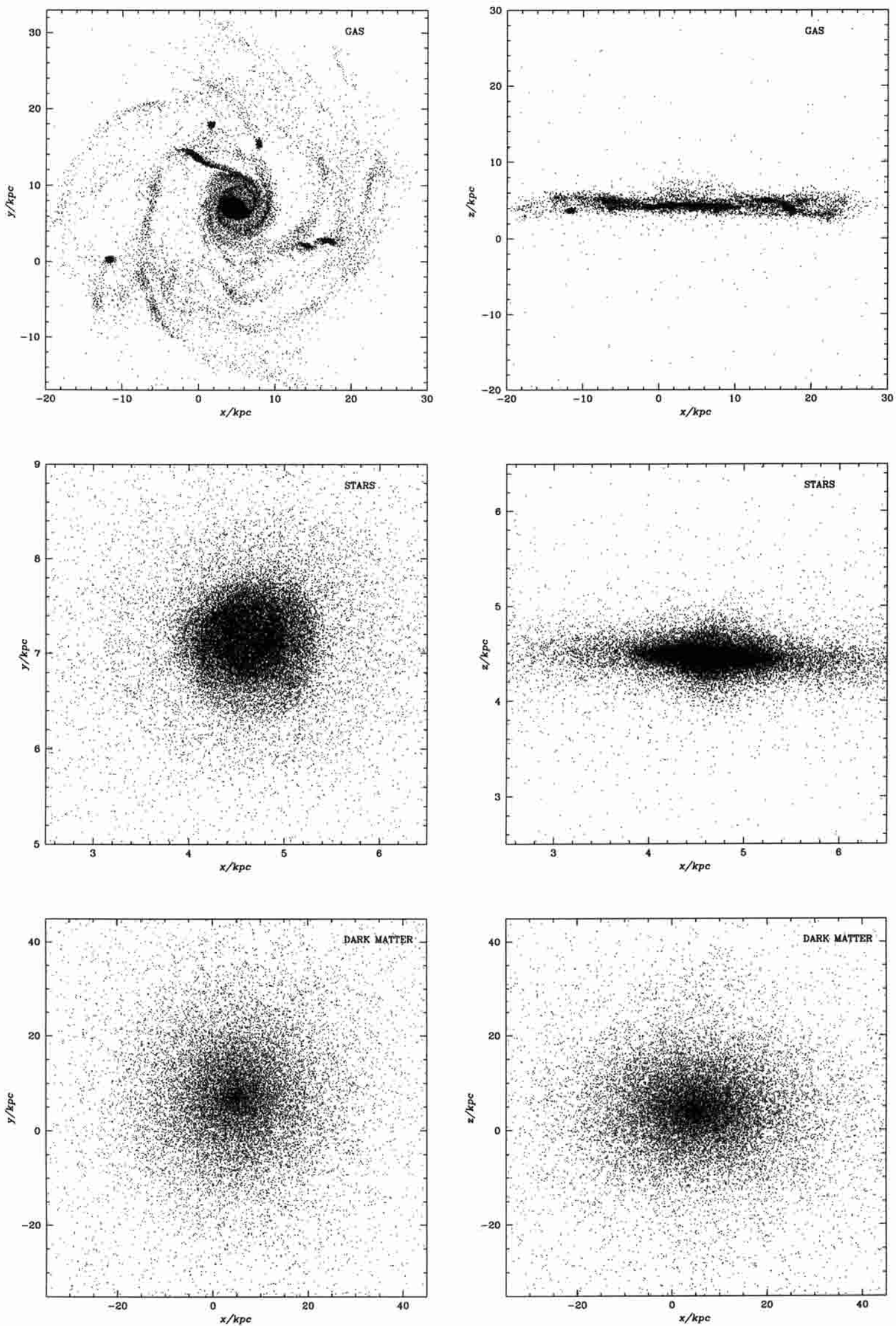

Fig. 5. $t_{\text {sim. }}=7.949$ Gyr. Note the reduced scale in the stellar images. 

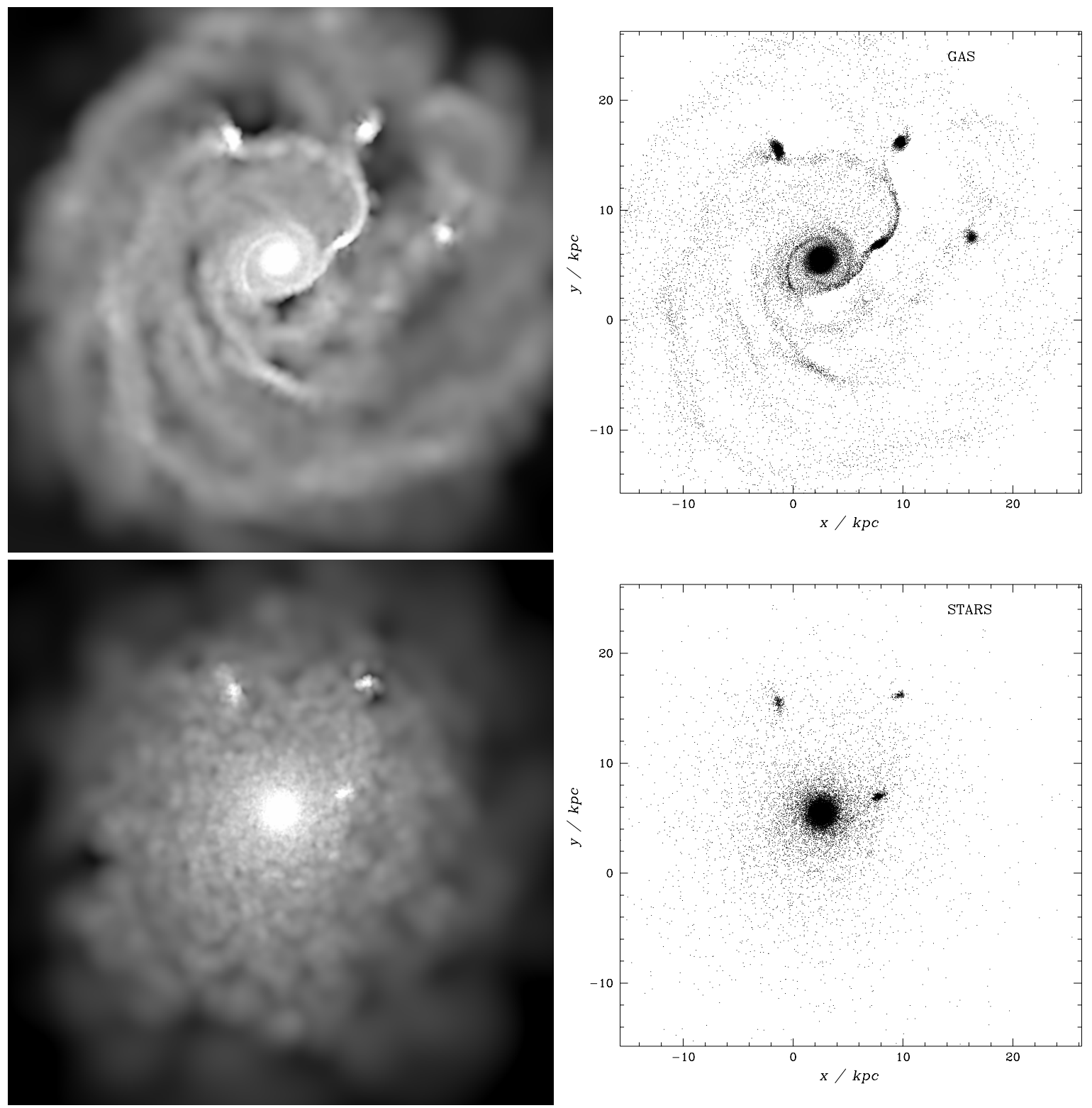

Fig. 6. The gas and stellar mass components, top and bottom respectively, at $t_{\text {sim. }}=8.570$ Gyr. In the smoothed plot, black and white regions have mass densities of less than $0.015 M_{\odot} \mathrm{pc}^{-2}$ and greater than $280 M_{\odot} \mathrm{pc}^{-2}$ respectively. These images were produced by calculating smoothed mass surface densities using SPH, evaluated on a grid of points. SPH kernel radii $h_{\mathrm{i}}$ were chosen such that 50 particles were contained within $2 h_{\mathrm{i}}$. The images show a region $42 \times 42 \mathrm{kpc}$, and $480 \times 480$ points were used. Note that the mass surface densities of gas, stars, and stellar remnants in the Solar neighbourhood are roughly 5,26 and $18 M_{\odot} \mathrm{pc}^{-2}$ respectively (Binney \& Tremaine 1987). At this time, $94 \%$ of the star mass was found to be older than $10^{8}$ yr. Thus the gas image is a better indicator of recent star formation, while the stellar image is comparable to an infra-red observation. Note that the mottled effect in the stellar plot is due to the fact that the image is constructed from particles.

During this phase the star formation rate increased sharply from $0.02 M_{\odot} \mathrm{yr}^{-1}$ to $4.3 M_{\odot} \mathrm{yr}^{-1}$, and roughly $5 \times 10^{8} M_{\odot}$ of gas was converted into stars over a timescale of 3.2 Gyr. For the duration of most of the collapse, the star formation remained roughly evenly distributed throughout the proto-galactic cloud. However by $t_{\text {sim. }} \simeq$ 6.2 Gyr, Fig. 3, star formation had begun to become more concentrated within discrete peaks in the gas density distribution.

\subsection{Phase III: Re-expansion and fragmentation $\left(t_{\text {sim. }} \simeq 6.2\right.$ Gyr to $\left.t_{\text {sim. }} \simeq 6.7 \mathrm{Gyr}\right)$}

The approximately spherical re-expansion of the dark matter component allowed the re-expansion of the gas disc in the horizontal direction due to conservation of angular momentum, see Figs. 3 and 4. During this phase the gas component fragmented into distinct high 

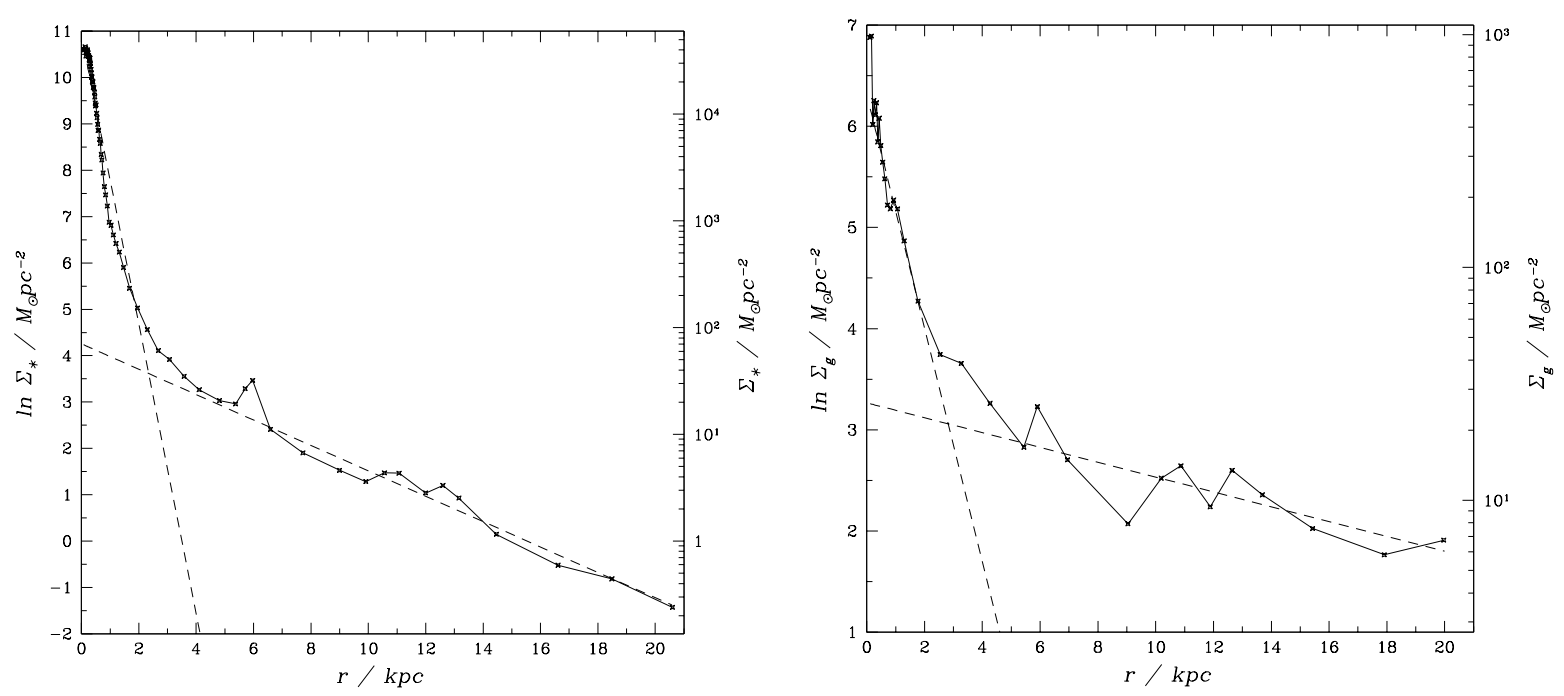

Fig. 7. The stellar surface density and gas stellar surface density profiles, taken at $t_{\text {sim. }}=8.570$ Gyr, Fig. 6 . The profiles were calculated by summing up the total mass in concentric annulli centred on the numerical galaxy's geometric centre such that each annulli contained roughly 1000 particles. Least squares fits of exponential functions to the bulge and disk regions are shown with dashed lines, i.e. $\Sigma_{\mathrm{g}}(r)=\Sigma_{0, \mathrm{bg}} \exp \left[-r / \alpha_{\mathrm{bg}}\right]+\Sigma_{0, \mathrm{dg}} \exp \left[-r / \alpha_{\mathrm{dg}}\right]$ and $\Sigma_{*}(r)=\Sigma_{0, \mathrm{~b}} \exp \left[-r / \alpha_{\mathrm{b}}\right]+\Sigma_{0, \mathrm{~d}} \exp \left[-r / \alpha_{\mathrm{d}}\right]$. The small density peaks observed at $r / \mathrm{kpc}=6,11$, and 13 in both graphs are due to the three remaining sub-galaxies, see Fig. 6 .
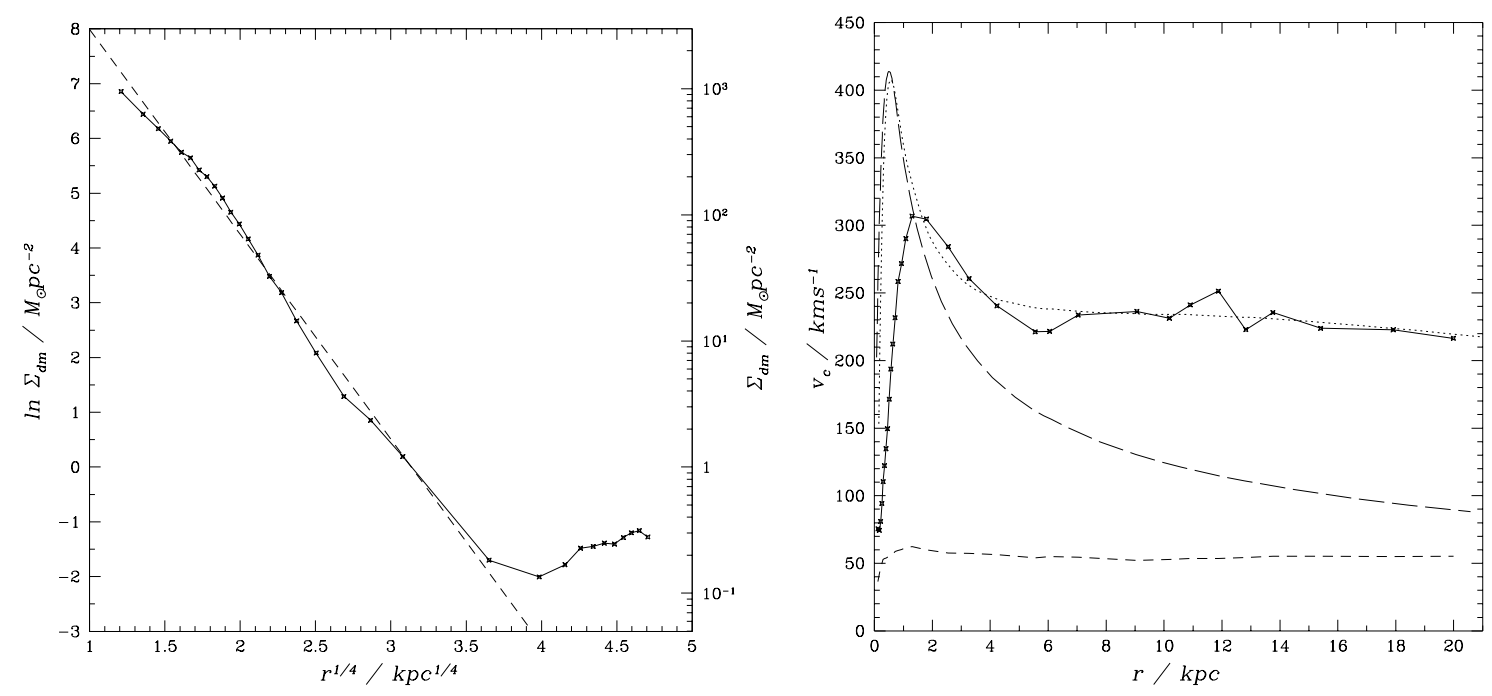

Fig. 8. The dark matter surface density profile and rotation curve, taken at $t_{\text {sim. }}=8.570$ Gyr, Fig. 6. These profiles were calculated in a similar way to the gas and stellar surface density profiles discussed above. The least squares fit of a de Vaucouleurs profile, i.e. $\Sigma_{\mathrm{dm}}(r)=\Sigma_{0, \mathrm{dm}} \exp \left[-\left(r / \alpha_{\mathrm{dm}}\right)^{1 / 4}\right]$ to the dark matter profile is shown with a dashed line. In the right graph the mean gas circular velocity is shown with a solid line. The circular velocity associated with the total, gas, and stellar mass within a given radius are shown with dotted, short dashed, and long dashed lines respectively (i.e. $v_{\mathrm{c}}^{2}=m(<r) / r$ where $m(<r)$ is the mass within spherical radius $r$ ).

density objects, which subsequently formed individual stellar components.

The central region of smooth dark matter density grew as successive shells of mass fell onto the central halo. This process was complete once all dark matter had passed through the central regions, and had either become part of the smooth featureless central halo (via violent relaxation), or continued to expand outwards in radius, see Fig. 3. The low density outer regions of the dark matter distribution, which contained roughly $30 \%$ of the dark matter, had not undergone full violent relaxation, so that remnants of the initial configuration were still visible. Subsequent to this re expansion, the parameters describing the dark matter surface density profile exhibited a damped oscillatory behaviour, which can be interpreted physically as a damped radial pulsation in the dark matter halo. Only two periods of oscillation were observed before the dark matter halo settled to an equilibrium configuration over the region in which the luminous galaxy components would eventually form and evolve, see Fig. 13.

The re-expansion of the dark matter allowed the gas mass which had experienced high densities at maximum 

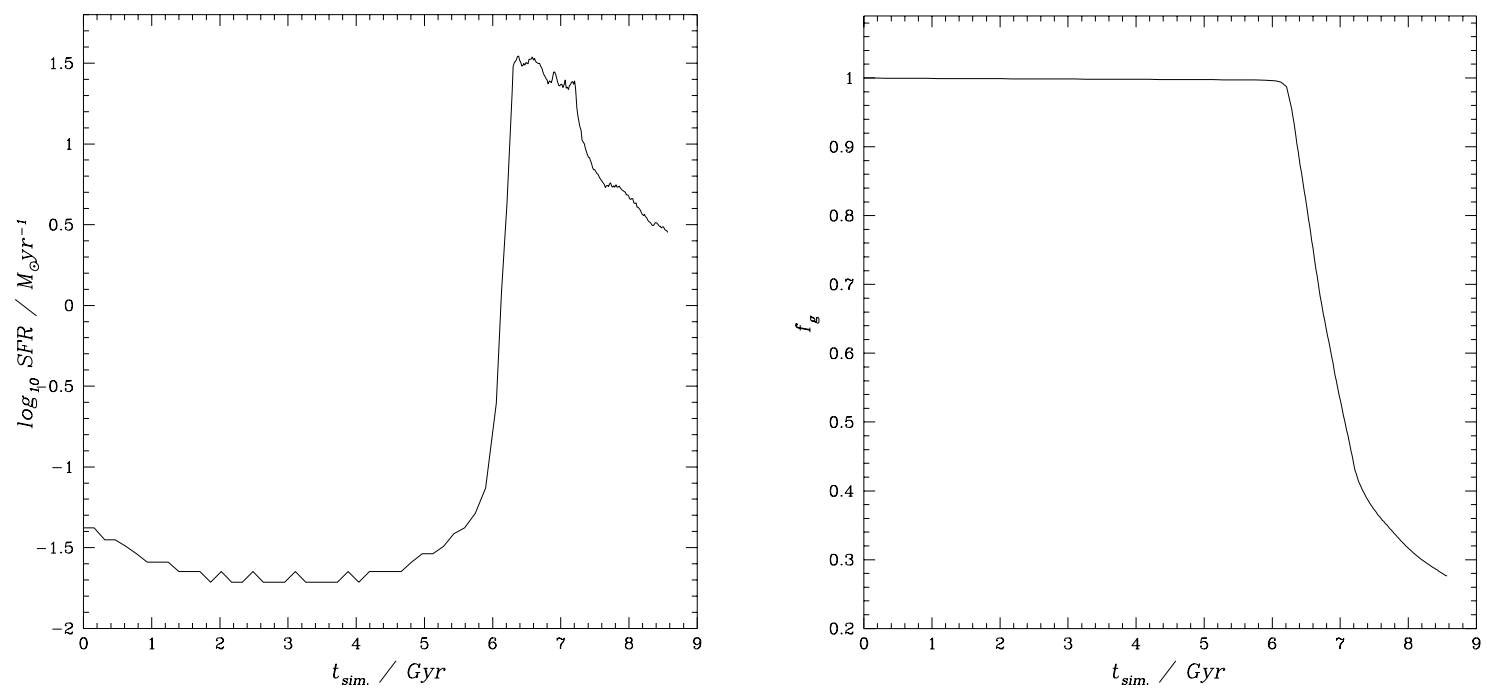

Fig. 9. The global star formation rate SFR and the gas mass fraction $f_{\mathrm{g}}$ versus time $t_{\text {sim. }}$. The star formation rate was calculated as the sum of the instantaneous star formation rate of all gas particles i.e. SFR $=\sum k_{\mathrm{s}} m_{\mathrm{i}} \rho_{\mathrm{i}}^{n_{\mathrm{s}}-1}$ where $m_{\mathrm{i}}$ and $\rho_{\mathrm{i}}$ are the mass and density of the $i$ th gas particle respectively, and $k_{\mathrm{s}}$ and $n_{\mathrm{s}}$ are the Schmidt law parameters discussed in Sect. 2.1.
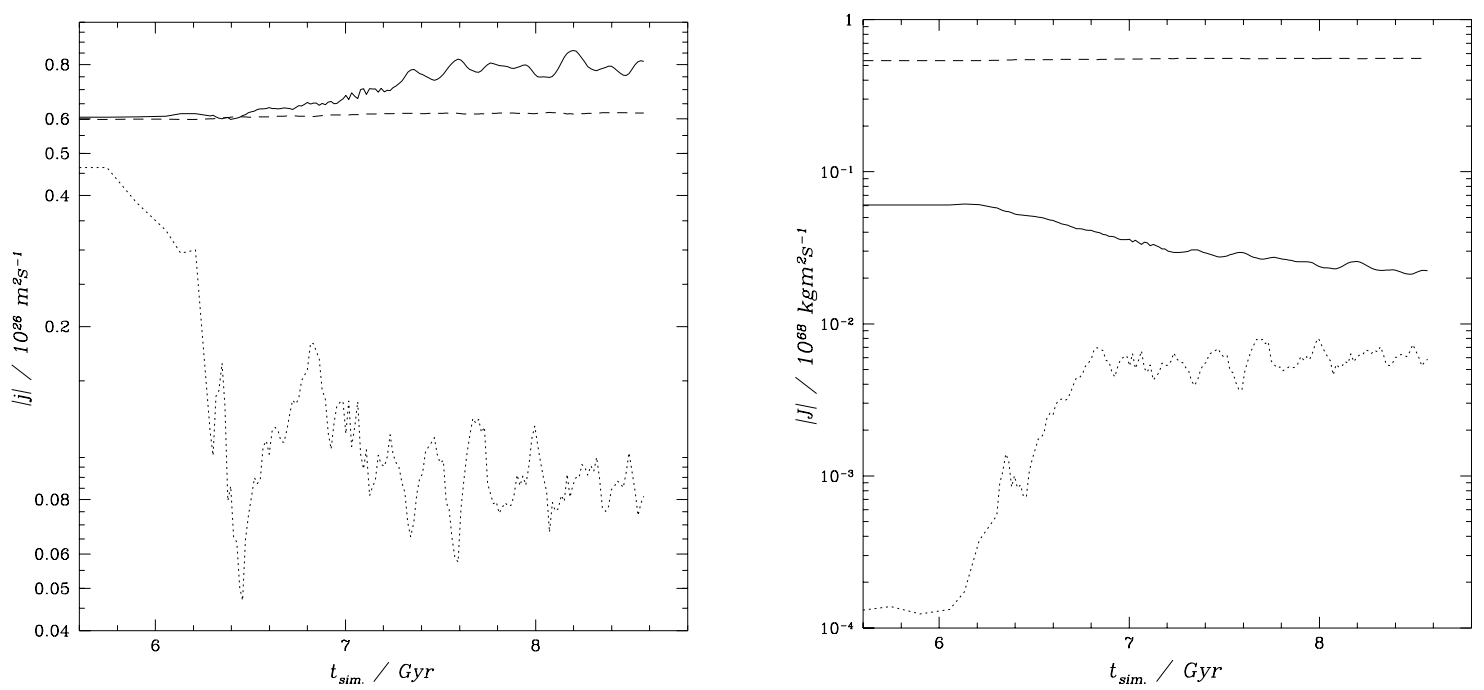

Fig. 10. Total specific angular momentum $|j|$ and total angular momentum $|J|$, versus time $t_{\text {sim. }}$. The angular momenta for each of the gas, stellar, and dark matter components are shown with solid, dotted, and dashed lines respectively. The total angular momentum was conserved to less than $0.1 \%$ during this simulation.

collapse to also re-expand. This gas, having radiatively dissipated away most of its energy in motion perpendicular to the proto-galactic disk, could only centrifugally re-expand in the horizontal plane. This outwardly moving ring of mass collided and shocked into the infalling gas, and began to fragment. Radial growth of the overall gas disk continued due to infall from the gaseous halo. The disk height was found to be roughly $1 \mathrm{kpc}$, but slowly became thinner as viscous forces damped out oscillations perpendicular to the proto-disk. The gas mass at this time, just after $t_{\text {sim. }} \simeq 6.2$ Gyr, Fig. 3 , was found to reside in three different environments: dense clumps, a highly inhomogeneous and geometrically complex inter-clump medium, and a smooth distribution of infalling gas from the low density gaseous halo. The formation of the dense clumps occurred through localized gravitational collapse within the proto-galactic disk. Gas mass in the centres of these clumps had a density of typically $\rho_{\mathrm{g}}=1 \mathrm{M}_{\odot} \mathrm{pc}^{-3}$, implying a Jeans length $\lambda_{\mathrm{j}}$ of

$\lambda_{\mathrm{j}}=\left(\frac{\pi c_{\mathrm{s}}^{2}}{G \rho_{\mathrm{g}}}\right)^{1 / 2} \simeq 200\left(\frac{\rho_{\mathrm{g}}}{1 M_{\odot} \mathrm{pc}^{-3}}\right)^{-1 / 2} \mathrm{pc}$

where the velocity dispersion of small clouds $c_{\mathrm{s}}=$ $7.5 \mathrm{~km} \mathrm{~s}^{-1}$ and $G$ is Newton's constant. The gas in the surrounding tenuous media was roughly one to two orders of magnitude lower. These dense gas clumps quickly developed into distinct objects with associated star formation, see Fig. 4, although they did not have distinct dark matter halos. At this time in the evolution of the numerical proto-galaxy, it is a matter of definition as to whether the numerical proto-galaxy should be considered as a single unified object, or as a collection of small galaxy-like 

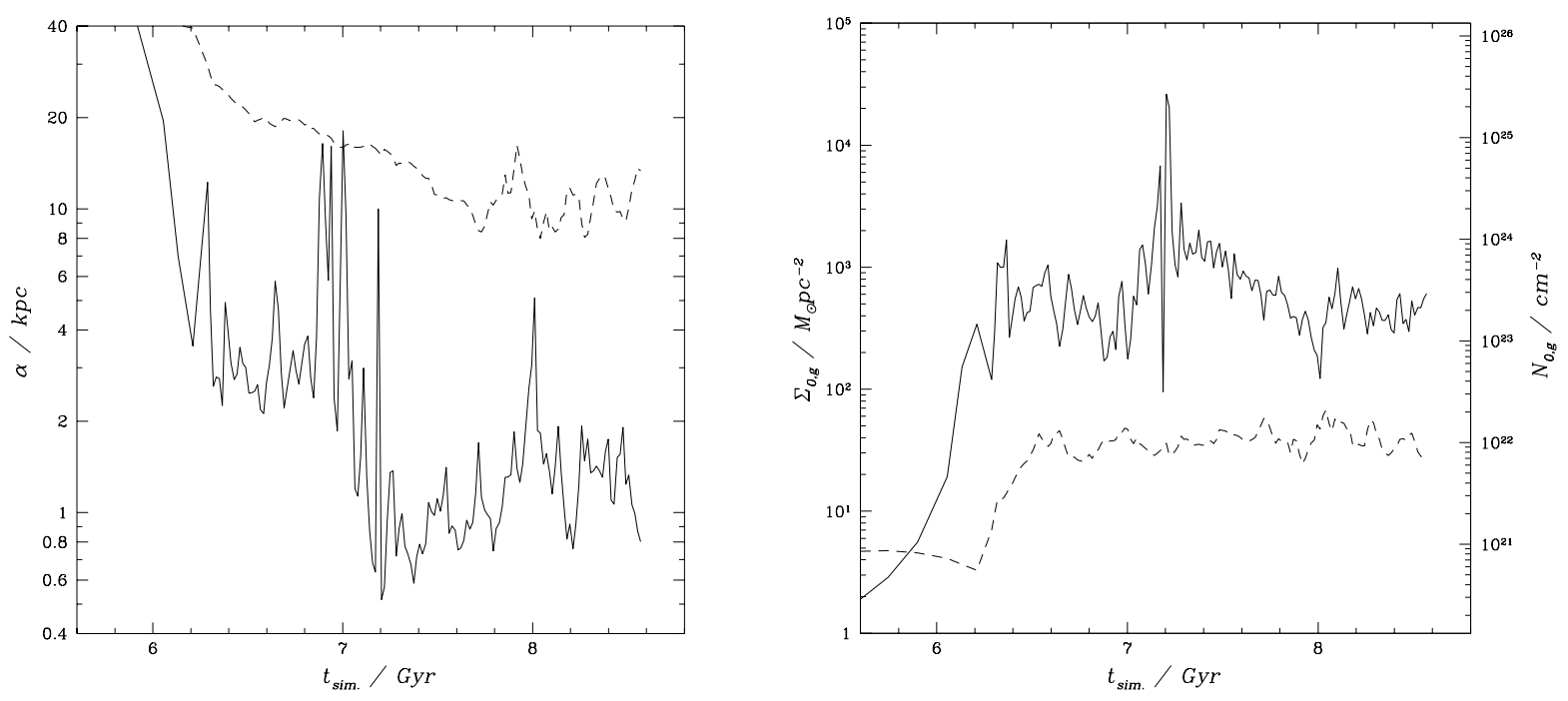

Fig. 11. Gas disk and gas pseudo-bulge scale lengths $\alpha_{\mathrm{dg}}$ and $\alpha_{\mathrm{bg}}$, and the gas disk and gas pseudo-bulge central surface densities $\Sigma_{0, \mathrm{dg}}$ and $\Sigma_{0, \mathrm{bg}}$ versus time $t_{\mathrm{sim}}$. See the caption of Fig. 7 for definitions of these parameters. In both graphs, the solid and dotted lines refer to the bulge and the disk fitting parameters respectively.
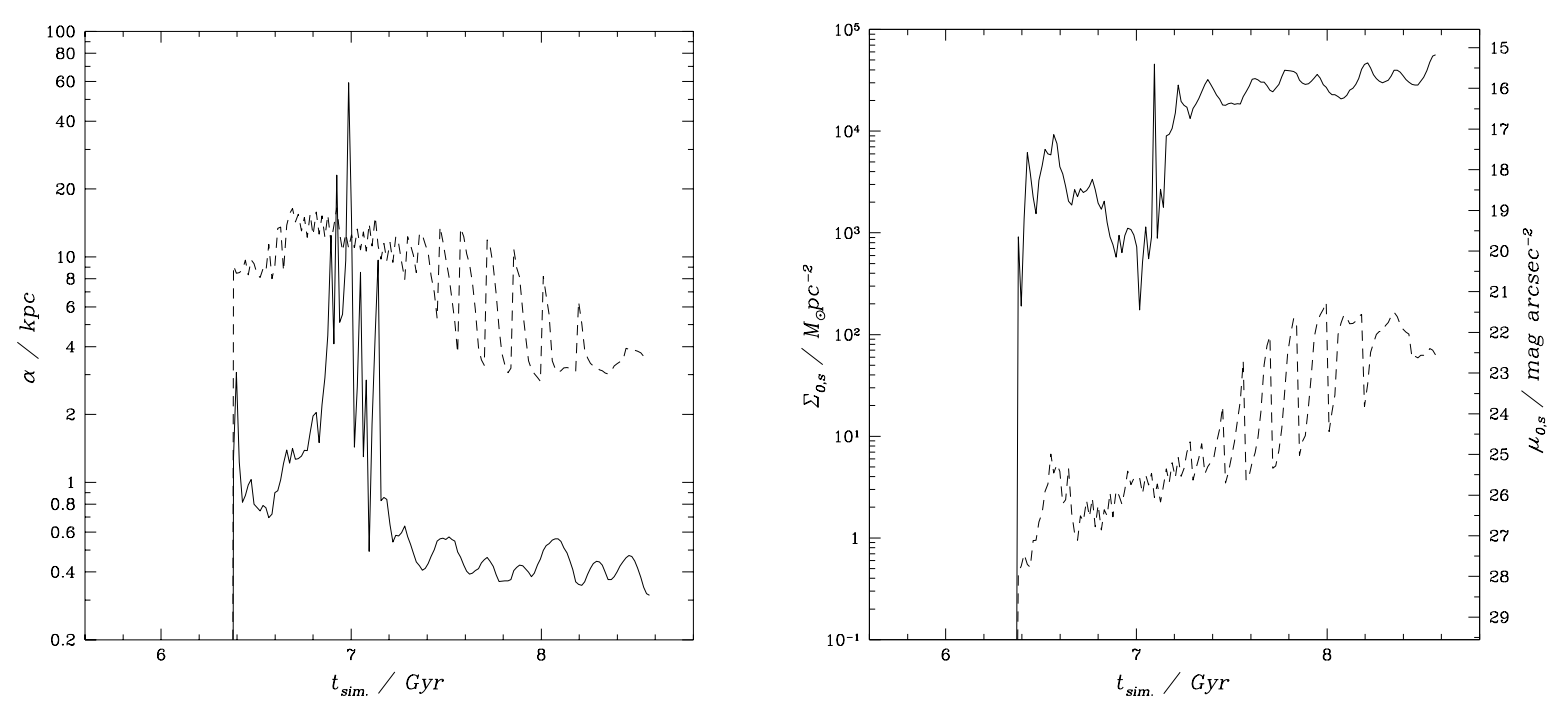

Fig. 12. Stellar disk and stellar bulge scale lengths $\alpha_{\mathrm{d}}$ and $\alpha_{\mathrm{b}}$, and gas disk and gas bulge central surface densities $\Sigma_{0, \mathrm{~d}}$ and $\Sigma_{0, \mathrm{~b}}$, versus time $t_{\mathrm{sim}}$. See the caption of Fig. 7 for definitions of these parameters. In both graphs, the solid and dashed lines refer to the bulge and disk fitting parameters respectively. Surface brightnesses were calculated using a mass-to-light ratio $\mathcal{M} / \mathcal{L}=1 M_{\odot} / L_{\odot}$. Thus, the correction to the given surface brightnesses for a preferred mass-to-light ratio $\mathcal{M} / \mathcal{L}$ is $+2.5 \log _{10} \mathcal{M} / \mathcal{L}$.

objects. In the following discussion of these objects, they will be referred to as "sub-galaxies", see Figs. 14 and 15.

Due to the highly clumpy nature of the gas during this phase, the geometric centre, as defined by the position of the largest central clump, was often significantly offset from the centre of mass, which resulted in large fluctuations in the fitting parameters describing the overall surface density of the proto-disk, see Figs. 11 and 12. The disk surface density profiles were affected to a lesser extent than the bulge profiles, since the outer regions were less sensitive to the choice of geometric centre, and the outer radial bins were azimuthally averaged over a larger area. Note that both the stellar and gas disk surface density profiles had begun to evolve in a stable fashion by the end of this phase (with the exception of the glitch between 7 and 7.2 Gyr, which will be discussed in more detail in the following section).

By $t_{\text {sim. }}=6.7$ Gyr, Figs. 4, 6 main sub-galaxies had formed, with masses in the range $5.9 \times 10^{8}$ to $1.6 \times$ $10^{10} M_{\odot}$, and major axes from 2 to $3.25 \mathrm{kpc}$. Peak gas densities in these evolved sub-galaxies were found to be of order 10 to $100 M_{\odot} \mathrm{pc}^{-3}$, a factor of roughly $10^{3}$ higher than the surrounding material, and a factor of roughly $10^{8}$ higher than the extended gaseous halo. These values compare to a total gas density of $0.042 M_{\odot} \mathrm{pc}^{-3}$ in The Galaxy at the Solar radius (Binney \& Tremaine 1987). The tidal torques induced through the non-circular orbits of the sub-galaxies caused their peak density to 

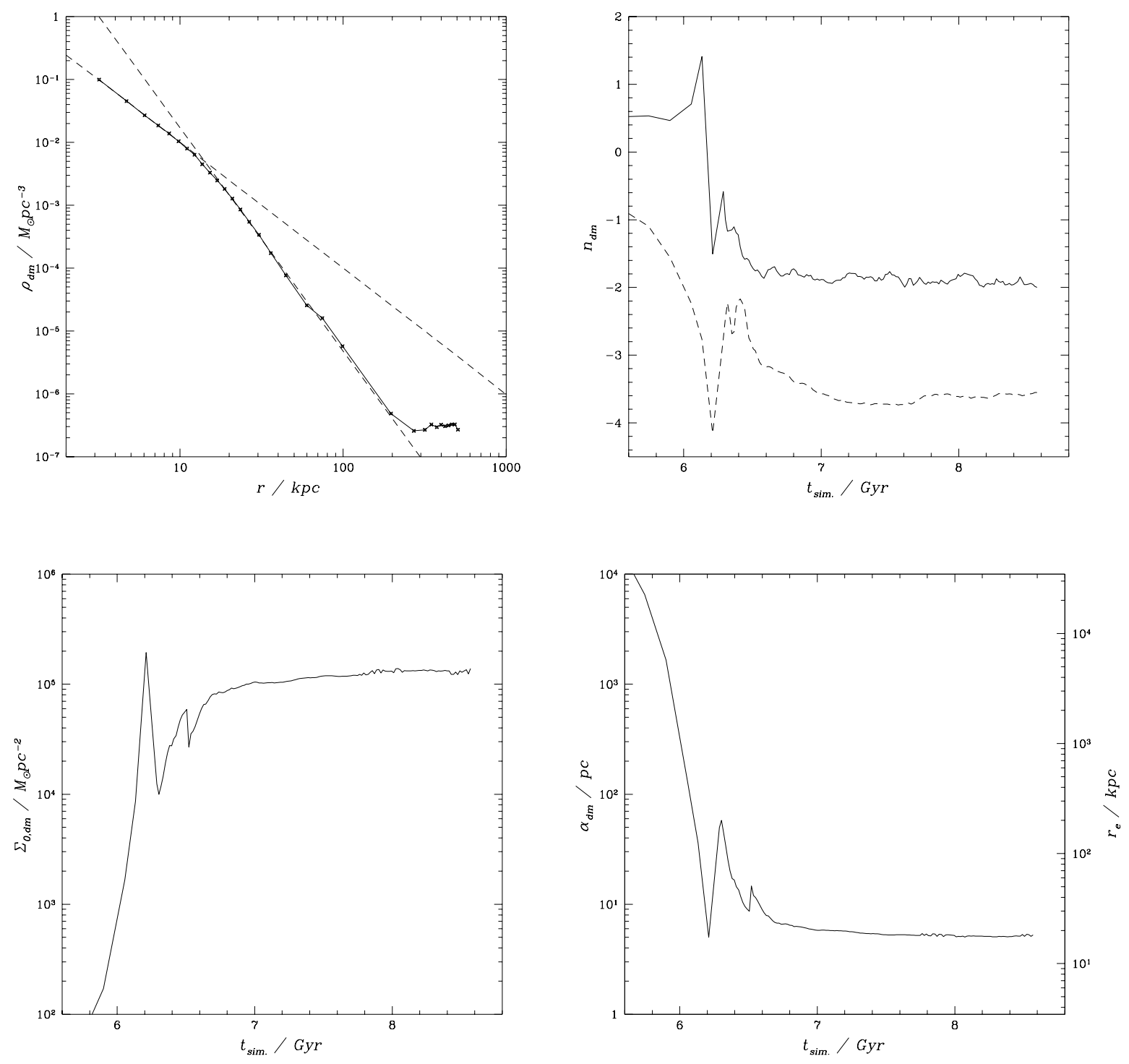

Fig. 13. The dark matter volume density profile, taken at $t_{\text {sim. }}=8.570$ Gyr, Fig. 6 , and a graph of the power law indices characterizing the dark matter volume density profile $n_{\mathrm{dm}}$ versus time $t_{\mathrm{sim}}$. (top); and the dark matter central surface density $\Sigma_{0, \mathrm{dm}}$ and the de Vaucouleurs scale length $\alpha_{\mathrm{dm}}$, versus time $t_{\mathrm{sim}}$. (bottom), see the captions of Fig. 8 and Table 2 for definitions of these parameters. In the top plots least squares fits of a power law density profile, i.e. $\rho_{\mathrm{dm}}(r) \propto r^{n_{\mathrm{dm}}}$, to the inner and intermediate regions of the dark matter density profile are shown with dotted lines, and the evolution of the inner and intermediate regions' indices are shown with solid and dotted lines respectively.

periodically fluctuate. The fraction of the total mass of each of the sub-galaxies in gaseous form varied from 0.18 to 0.78 , the heaviest generally having the lowest gas mass fractions, the highest mean densities, and the highest star formation rates. The sub-galaxies were found to be supported by a combination of rotation and velocity dispersion. Their orbits were frequently found to be inclined to the plane of the proto-disk, but typically at inclination angles less than $15^{\circ}$, see Fig. 4 . The linear sizes and masses of these sub-galaxies are similar to those inferred from the clumps of luminosity observed (sometimes with apparently linear distributions) in some of the Hubble Deep Field galaxies (Williams et al. 1996; van den Bergh et al. 1996; Abraham et al. 1994; Mobasher et al. 1996; Clements \& Couch 1996); and a distribution of clumpy star forma- tion, roughly confined to a disk would appear as a chainlike structure if the disk were viewed edge-on.

During this phase the global star formation rate fell to roughly $27 M_{\odot} \mathrm{yr}^{-1}$ after reaching a peak of $35 M_{\odot} \mathrm{yr}^{-1}$ during the formation of the sub-galaxies. Roughly $32 \%$ of the initial gas mass, $1.6 \times 10^{10} M_{\odot}$, was converted to stellar material over a timescale of $5 \times 10^{8} \mathrm{yr}$. During this phase the ratio of gas mass inside of the sub-galaxies to that outside was roughly 0.2 , while the ratio of typical gas densities inside to that outside was $10^{3}$. Therefore, the star formation rate densities inside the sub-galaxies were typically a factor of $\left(10^{3}\right)^{1.5}=10^{4.5}$ higher than those outside: during this epoch star formation activity was concentrated in the sub-galaxies. 

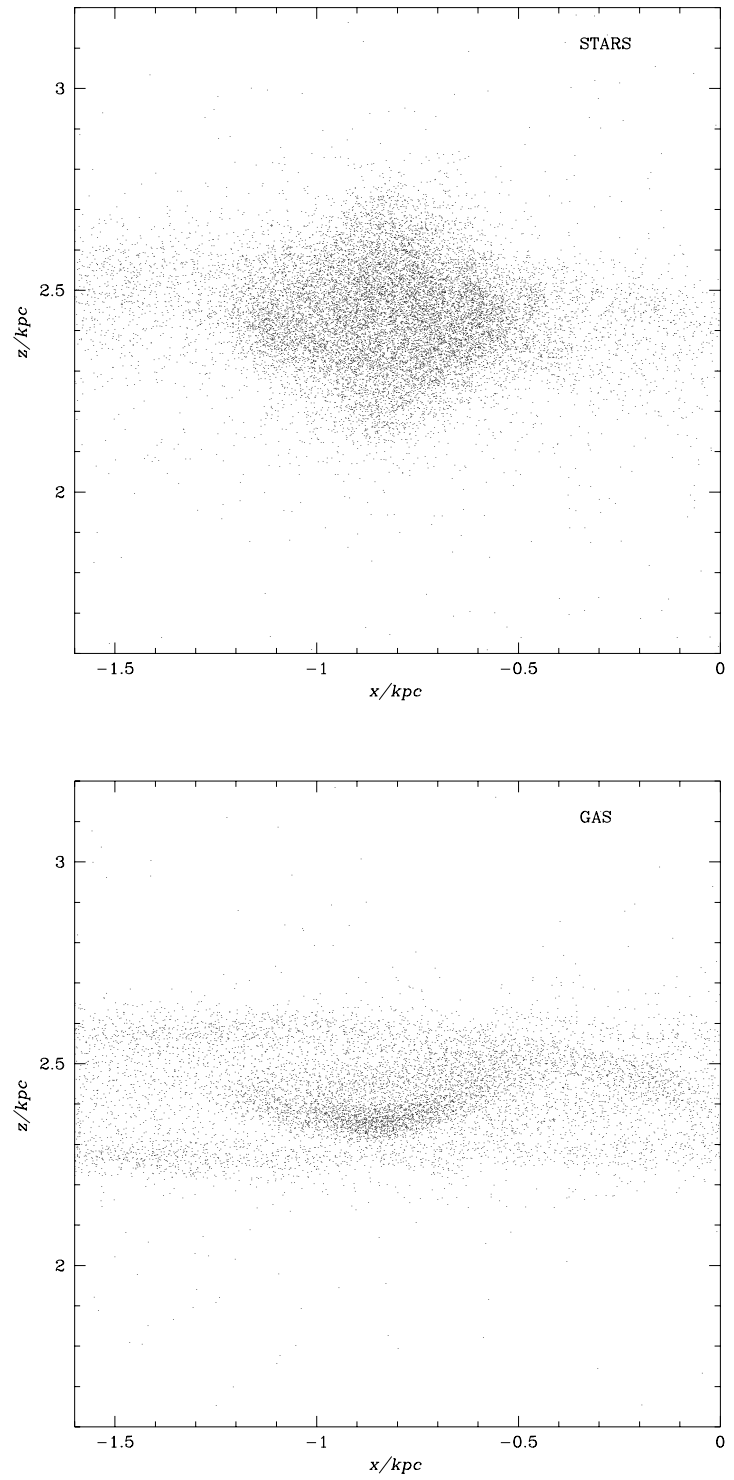

Fig. 14. The central sub-galaxy, taken at $t_{\text {sim. }}=6.8$ Gyr.

\subsection{Phase IV: The smoothing of inhomogeneity in the proto-disk $\left(t_{\text {sim. }} \simeq 6.7\right.$ Gyr to $\left.t_{\text {sim. }} \simeq 7.9 \mathrm{Gyr}\right)$}

By $t_{\text {sim. }}=6.7$ Gyr disruption of the sub-galaxies by tidal torques, the progressive circularization of gas and star particle orbits, and the removal of inhomogeneity in the gas and stellar density distributions had begun. This was the most dynamically active epoch in the numerical galaxy's evolution. The tidal disruption of the sub-galaxies resulted in shocks and linear features in the gas, as well as arcs of stellar mass around the central sub-galaxy, see Figs. 4 and 5. This stellar mass was formed in the subgalaxies before their disruption, and was not associated with the high density gas found in the shocked regions formed.
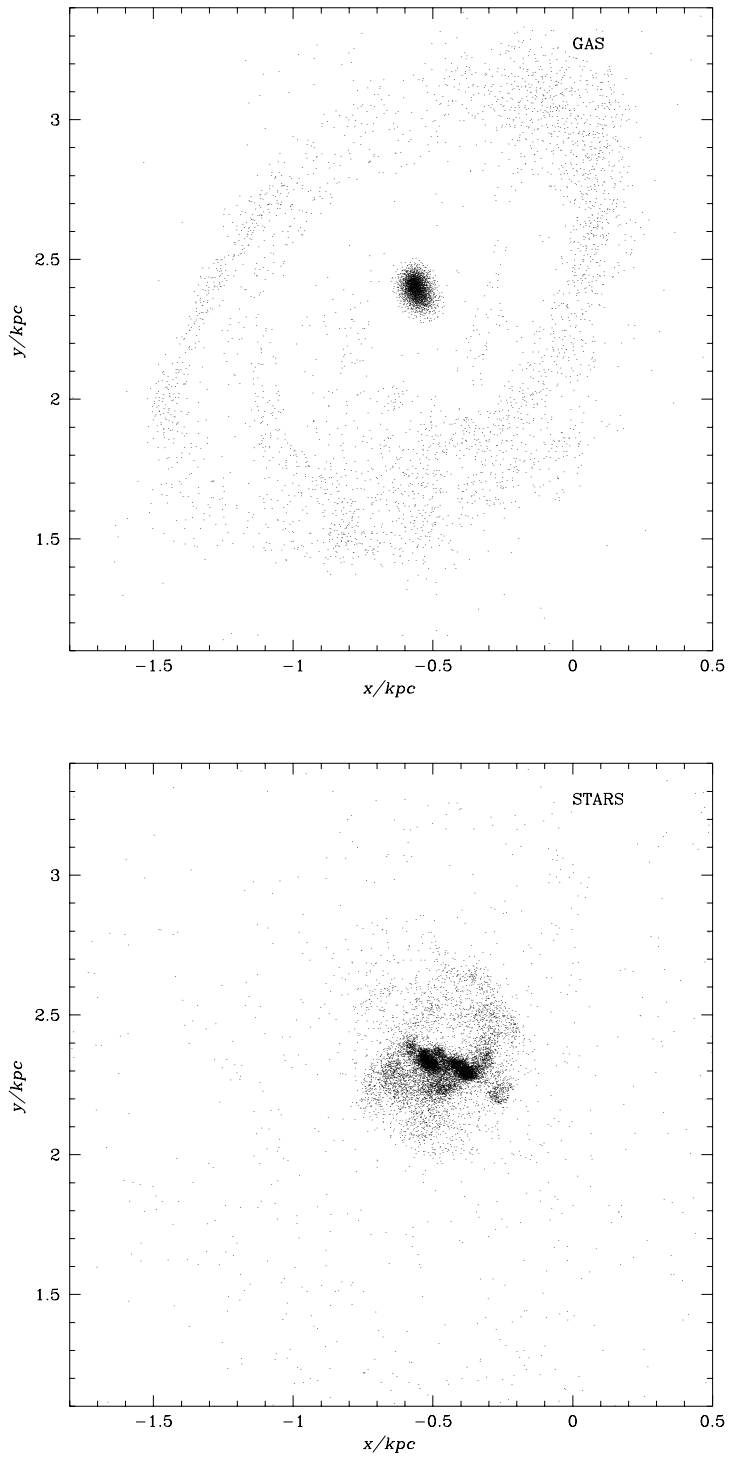

Fig. 15. The highest density sub-galaxy, taken at $t_{\text {sim. }}=$ 6.8 Gyr.

The evolution during this phase was dominated by the merging of the two most massive and most bound sub-galaxies. Firstly there is the proto-bulge type object at the centre of the galaxy, see Fig. 14. This sub-galaxy's morphology was that of a miniature disk galaxy, consisting of a thin gas disk, a thicker stellar disk and stellar spherical bulge, and contained some of the oldest stellar mass in the numerical galaxy formed in the early post-collapse phase. At $t_{\text {sim. }}=7 \mathrm{Gyr}$ this sub-galaxy had a total mass of $1.6 \times 10^{10} M_{\odot}$, a gas fraction of 0.18 , and a central peak gas density of roughly $500 M_{\odot} \mathrm{pc}^{-3}$. This object was the least bound of the two sub-galaxies in question, due to its comparatively large size. The second sub-galaxy, see Fig. 15, condensed out of the low density gas at large radii in the proto-disk (see Fig. $4,(x / \mathrm{kpc}, y / \mathrm{kpc}) \simeq(-6,0)$, the core/ring gas object), and thus spent a long time away 
from disruptive torques early in its evolution, which allowed the sub-galaxy to become sufficiently bound to evolve its own stellar component. Subsequent tidal interactions induced the gas in the sub-galaxy's central regions to very high densities, at times greater than $10^{3} M_{\odot} \mathrm{pc}^{-3}$, and correspondingly high star formation rates. This sub-galaxy was dwarf irregular in morphology, and obtained a gas ring by disrupting a smaller mass sub-galaxy while orbiting the central sub-galaxy. During this encounter, the sub-galaxy underwent significant gas infall into its central regions, and became very compact. The sub-galaxy's total mass and gas mass fraction were $4.5 \times 10^{9} M_{\odot}$ and 0.32 respectively. The eventual merger of this with the proto-bulge type object resulted in the destruction of the latter, see the animation to be found at http://www.astro.cf .ac.uk/pub/Alistair.Nelson/ indexgf.html. This event induced high star formation rates in the central regions, and spread the stellar component of the central sub-galaxy into a thick disk structure. The interaction induced strong shocks and spiral arms in the gas and stellar matter, however the nucleus of the second sub-galaxy was sufficiently bound to remain intact during the interaction, and inherited the position of numerical galaxy centre. Peak gas densities increased by roughly an order of magnitude during this interaction.

The parameters describing the stellar disk and gas disk evolved steadily during this phase: scale lengths showing only a slight decrease, see Figs. 11 and 12 . In the gas disk, this reflects the growth of the disk through infall, and the decrease in the ratio between the inner and outer disk surface density. As a consequence, star formation activity was weighted less towards the inner disk regions as the disk grew, and thus the scale length of the stellar disk increased. The gas disk central surface density remained roughly constant after this time. This can be interpreted as a balance between star formation, which tends to flatten the gas profile, and therefore lowers the central surface density; and infall of gas onto the disk, which increases the gas density of the disk. The stellar central surface density increased during this phase, reflecting the vigorous star formation activity present in the numerical galaxy disk during this epoch. The parameters describing the stellar bulge and central gas regions were highly variable during this phase, since during the merger of the two largest subgalaxies these fitting functions used were not appropriate descriptors of the bulge mass distribution, which accounts for the glitches at $t \simeq 7$ Gyr in Figs. 11 and 12 .

The global star formation rate decreased from $27 M_{\odot} \mathrm{yr}^{-1}$ to $6 M_{\odot} \mathrm{yr}^{-1}$ over a period of $1.2 \times 10^{8} \mathrm{yr}$. This decrease was generally smooth, but punctuated with small peaks, associated with the tidally induced star formation activity in the sub-galaxies. This was especially true during the merger of the two largest sub-galaxies. During this phase, $1.5 \times 10^{10} M_{\odot}$ of gas mass was converted into stellar mass, a similar amount to that converted during the previous phase, but over a duration of time twice as long.

\subsection{Phase V: A quiescent spiral galaxy (from $t_{\text {sim. }} \simeq 7.9 \mathrm{Gyr}$ )}

By this time, the numerical galaxy had stabilized: any further variations in morphology or structural parameters were small, and occurred on long timescales, see Figs. 5 and 6 . The remaining three sub-galaxies orbited on almost circular trajectories with shallow angles to the plane of the gas disk, thus they were long lived and difficult to disrupt by tidal interactions. Close tidal interactions between the central mass concentration and the remaining sub-galaxies were found to induce periodic and long-lived spiral structure in the central regions. During the later stages of this phase, one sub-galaxy survived for 3 orbits of period roughly $220 \mathrm{Myr}$, before interacting with another sub-galaxy. This resulted in the first sub-galaxy being slung-shot out into an eccentric orbit. The second sub-galaxy adopted an orbit similar to that of the first, and induced further periodic shocks in the inner gas disk. This continued for a further 3 orbits of similar period, i.e. a gravitational exchange event took place, see Fig. 5 where the two sub-galaxies are at $(x / \mathrm{kpc}, y / \mathrm{kpc}) \simeq(0,12)$ and $(x / \mathrm{kpc}, y / \mathrm{kpc}) \simeq(1,17)$, and then Fig. 6 where they are at $(x / \mathrm{kpc}, y / \mathrm{kpc}) \simeq(-2,16)$ and $(x / \mathrm{kpc}, y / \mathrm{kpc}) \simeq(8,6)$ respectively.

Shocks were found to be associated with the spiral structure prevalent during this phase, and were also observed in an adjoining arm between the sub-galaxy and the central stellar concentration. See Fig. 16, where the change in the direction of the gas flow post-shock and the induced spiral structure can be clearly seen. The density contrast between the pre-shock and post-shock gas was found to be roughly 6 . When the simulation was terminated at $\mathrm{t}_{\text {sim. }}=8.57 \mathrm{Gyr}$, Fig. 6, the gas disk had become smoother, although long-lived spiral structure in the central regions was still present. Only $7 \times 10^{9} M_{\odot}$ of gas remained to fall onto the disk, and the gas disk had thinned substantially to a height of less than 200 pc (compare Figs. 4 and 5).

The parameters describing the gas disk and bulge evolved steadily: the gas disk had a roughly constant central surface density and scale length, while the central regions of the gas profile had a decreasing central surface density and an increasing scale length, see Figs. 11 and 12. This can be interpreted as the central peak in gas surface density simultaneously flattening and decreasing in surface density due to star formation activity preferentially in the central regions. During this phase the parameters describing the stellar disk and bulge evolved systematically with an additional oscillatory behaviour, see Fig. 12. The systematic evolution involved slowly increasing central surface densities and decreasing scale lengths; while the oscillatory behaviour in the central surface densities, more prevalent in the disk parameters, was anti-correlated with those in the scale lengths. Such an evolution can be interpreted physically as a radial pulsation, with a period of roughly $200 \mathrm{Myr}$, similar to the rotational period in the numerical galaxy disk. The pulsations in the bulge and 


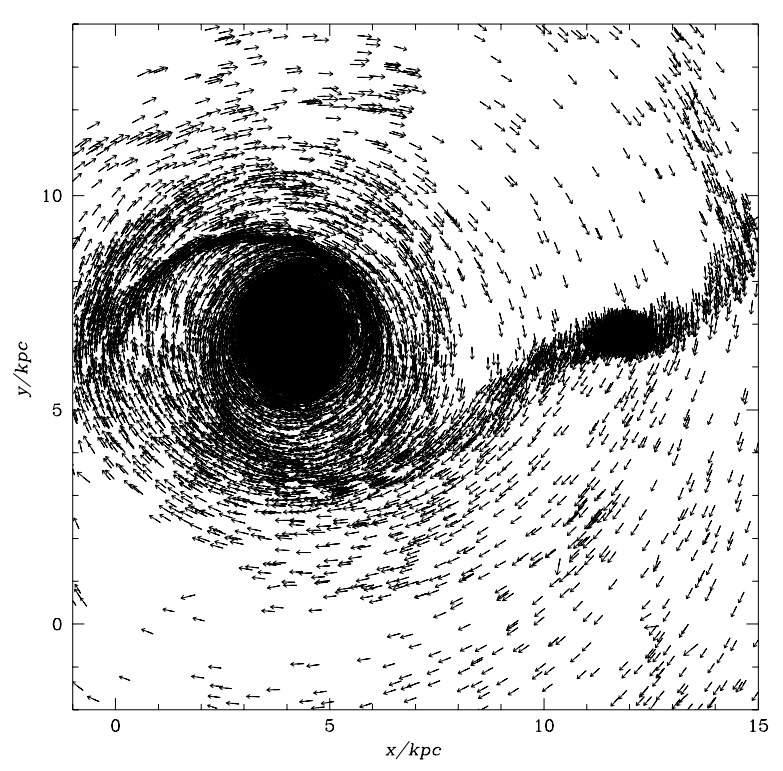

Fig. 16. A plot of gas particles with associated velocity vectors, taken at $t_{\text {sim. }}=7.825 \mathrm{Gyr}$, a comparable time to Fig. 5 . Note the characteristic radial elongation of the interacting subgalaxy, the change in direction of velocity vectors pre- and post-shock in the adjoining arm, and the induced spiral structure on the opposite side of the galaxy.

disk components were only roughly in phase, and were generally more regular in the disk region. It is notable that this behaviour began just after the merger of the two largest sub-galaxies.

The global star formation rate was found to decrease from $6 M_{\odot} \mathrm{yr}^{-1}$ to $3 M_{\odot} \mathrm{yr}^{-1}$ over a period of $1 \mathrm{Gyr}$. During this phase $4.7 \times 10^{9} M_{\odot}$ of gas mass was converted into stellar mass, resulting in a final stellar mass of $3.6 \times$ $10^{10} M_{\odot}(46016$ star particles were formed in total), and a total gas fraction of 0.28 . As the high star formation rates within the sub-galaxies decreased the peak in gas density at their centres, the star formation activity became much less concentrated within the sub-galaxies and was found to be more homogeneously spread through the galaxy disk.

The numerical galaxy's final parameters are summarized in Tables 1 and 2, and the final surface density profiles and rotation curve are shown in Figs. 7 and 8. The tabulated statistics indicate that the numerical galaxy is similar in gas fraction (Roberts \& Haynes 1994), star formation rate (Kennicutt 1997), disk size (Roberts \& Haynes 1994), bulge size (de Jong 1996a, 1996b), bulgeto-disk ratio (Simien \& de Vaucouleurs 1986; de Jong 1996a, 1996b), rotation curve (Casertano \& van Gorkom 1991; Persic \& Salucci 1995), gas surface density profile (Young \& Scoville 1991), and stellar surface density profile (de Jong \& van der Kruit 1994) to bright spiral galaxies in the local Universe such as The Galaxy and M 31. Based on this quantitative comparison, the Hubble type of this numerical galaxy may be estimated as Sbc.
Table 1. Global galaxy parameters of the numerical galaxy, taken at $t_{\text {sim. }}=8.570 \mathrm{Gyr}$, Fig. 6 . The properties given are: the global star formation rate SFR, the total gas fraction $f_{\mathrm{g}}$, the stellar bulge-to-disk mass ratio $B / D_{\mathrm{M}}$, the estimated stellar bulge-to-disk luminosity ratio $B / D_{\mathrm{L}}$, the bulge-to-total disk mass ratio $B / D_{\mathrm{M} \text {,tot., and the estimated bulge-to-total }}$

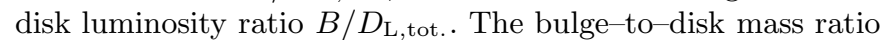
$B / D_{\mathrm{M}}=\Sigma_{0, \mathrm{~b}} \alpha_{\mathrm{b}}^{2} / \Sigma_{0, \mathrm{~d}} \alpha_{\mathrm{d}}^{2}$ and is the ratio of the total mass contained within each exponential profile. A lower limit on the

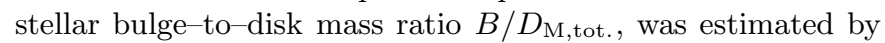
using the total disk mass calculated from the gas and stellar disk mass surface density profiles in place of the stellar disk mass in the above formula. The bulge-to-disk mass ra-

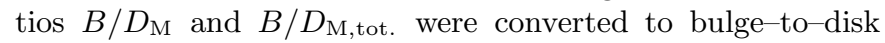
luminosity ratios $B / D_{\mathrm{L}}$ and $B / D_{\mathrm{L} \text {,tot. using the mass-to- }}$ light ratios $\mathcal{M} / \mathcal{L}_{V}=5 M_{\odot} / L_{\odot}$ (Charlot et al. 1996) for the older bulge stellar mass, and $\mathcal{M} / \mathcal{L}=0.67 M_{\odot} / L_{\odot}$ (Binney \& Merrifield 1998, p. 125) for the younger disk stellar mass.

\begin{tabular}{lc}
\hline Global Numerical Galaxy Properties & \\
\hline $\mathrm{SFR} / M_{\odot} \mathrm{yr}^{-1}$ & 3 \\
$f_{\mathrm{g}}$ & 0.28 \\
$B / D_{\mathrm{M}}$ & 5.9 \\
$B / D_{\mathrm{L}}$ & 0.8 \\
$B / D_{\mathrm{M}, \text { tot. }}$ & 1.0 \\
$B / D_{\mathrm{L}, \text { tot. }}$ & 0.1 \\
\hline
\end{tabular}

\section{Conclusions and discussion}

In summary, the main conclusions of this paper are:

1. The combination of simple models for star formation and the ISM and idealized proto-galactic initial conditions resulted in the formation of a numerical spiral galaxy with all of the gross properties of contemporary bright spiral galaxies.

2. This numerical galaxy was found to have a similar shaped rotation curve and similarly shaped mass surface density profiles to those observed. The measured parameters describing these profiles were found to be consistent with those of galaxies with Hubble type of roughly Sbc. Other features commonly observed in spiral galaxies, such as long lived spiral shocks, warps, and dwarf companions were also found. Such characteristics occurred naturally out of the physical processes modeled.

3. The evolution of this simulation suggested that the process of galaxy formation may be analysed into five phases which are characterized by the dominant processes occurring during each phase.

4. A new mechanism for the formation of stellar bulge components was suggested. The growth of the stellar bulge in this simulation was found to occur principally during a brief period of damped oscillation of gas about the plane of the proto-galactic disk. This was caused by the asymmetric, clumpy gas distribution on either side of the proto-galactic plane overshooting, and not instantaneously dissipating all kinetic energy perpendicular to the proto-galactic disk. 
Table 2. The structural parameters describing the numerical galaxy, taken at $t_{\text {sim. }}=8.570 \mathrm{Gyr}$, Fig. 6 . The parameters not defined elsewhere (see the captions of Figs. 7 and 8) are defined as: the radius at which the stellar bulge and disk fitting functions intersect $R_{\mathrm{b}}$; the radius of the $25 V-\mathrm{mag} \operatorname{arcsec}^{-2}$ isophote $R_{\mathrm{d}}$; the gas mass fraction inside of $R_{\mathrm{b}}, f_{\mathrm{b}, \mathrm{g}}$; and the gas mass fraction outside of $R_{\mathrm{b}}, f_{\mathrm{d}, \mathrm{g}}$. Stellar mass surface densities $\Sigma_{0, \mathrm{~b}}$ and $\Sigma_{0, \mathrm{~d}}$ were converted to luminosity surface densities $\Sigma_{0, \mathrm{~b}}^{L}$ and $\Sigma_{0, \mathrm{~d}}^{L}$ using the mass-to-light ratios given in the caption of Table 1, from which the surface brightnesses $\mu_{0, \mathrm{~b}}$ and $\mu_{0, \mathrm{~d}}$ were calculated. Gas surface densities $\Sigma_{0, \mathrm{bg}}$ and $\Sigma_{0, \text { dg }}$ are also given in terms of the equivalent column density of hydrogen atoms $N_{0, \mathrm{bg}}$ and $N_{0, \mathrm{dg}}$. The variable $r_{\mathrm{e}}$ is the half mass radius of the dark matter distribution, as defined by the fitted de Vaucouleurs law.

\begin{tabular}{|c|c|}
\hline \multicolumn{2}{|c|}{ Numerical Galaxy Parameters } \\
\hline \multicolumn{2}{|l|}{ Bulge: } \\
\hline$R_{\mathrm{b}} / \mathrm{kpc}$ & 2.3 \\
\hline$f_{\mathrm{b}, \mathrm{g}}$ & 0.045 \\
\hline$\alpha_{\mathrm{b}} / \mathrm{kpc}$ & 0.3 \\
\hline$\alpha_{\mathrm{bg}} / \mathrm{kpc}$ & 0.8 \\
\hline$\Sigma_{0, \mathrm{~b}} / 10^{3} M_{\odot} \mathrm{pc}^{-2}$ & 56 \\
\hline$\Sigma_{0, \mathrm{~b}}^{L} / 10^{3} L_{V, \odot \mathrm{pc}^{-2}}$ & 11 \\
\hline$\mu_{0, \mathrm{~b}} / V-\mathrm{mag} \operatorname{arcsec}^{-2}$ & 17 \\
\hline$\Sigma_{0, \mathrm{bg}} / 10^{3} M_{\odot} \mathrm{pc}^{-2}$ & 0.6 \\
\hline$N_{0, \mathrm{bg}} / 10^{22} \mathrm{~cm}^{-2}$ & 8 \\
\hline \multicolumn{2}{|l|}{ Disk: } \\
\hline$R_{\mathrm{d}} / \mathrm{kpc}$ & 10 \\
\hline$f_{\mathrm{d}, \mathrm{g}}$ & 0.6 \\
\hline$\alpha_{\mathrm{d}} / \mathrm{kpc}$ & 3.8 \\
\hline$\alpha_{\mathrm{dg}} / \mathrm{kpc}$ & 13 \\
\hline$\Sigma_{0, \mathrm{~d}} / M_{\odot} \mathrm{pc}^{-2}$ & 67 \\
\hline$\Sigma_{0, \mathrm{~d}}^{L} / L_{V, \odot} \mathrm{pc}^{-2}$ & 100 \\
\hline$\mu_{0, \mathrm{~d}} / V-\mathrm{mag} \operatorname{arcsec}^{-2}$ & 22 \\
\hline$\Sigma_{0, \mathrm{dg}} / M_{\odot} \mathrm{pc}^{-2}$ & 26 \\
\hline$N_{0, \mathrm{dg}} / 10^{22} \mathrm{~cm}^{-2}$ & 0.3 \\
\hline \multicolumn{2}{|l|}{ Dark Matter Halo: } \\
\hline$\Sigma_{0, \mathrm{dm}} / 10^{6} M_{\odot} \mathrm{pc}^{-2}$ & 0.1 \\
\hline$\alpha_{\mathrm{dm}} / \mathrm{pc}$ & 5.2 \\
\hline$r_{\mathrm{e}} / \mathrm{kpc}$ & 18 \\
\hline$v_{\mathrm{c}} / \mathrm{km} \mathrm{s}^{-1}$ & 230 \\
\hline
\end{tabular}

Before discussing these issues in more detail, it is appropriate to first establish the reliability and limitations of the numerical solution discussed here. The code was able to reproduce solutions to the Riemann shock tube (Hernquist \& Katz 1989), the evolution of a Plummer sphere of collisionless matter (Hernquist 1987; Binney \& Tremaine 1987), and gaseous accretion onto a point mass (Bertschinger 1985; Navarro \& White 1993), with accuracies comparable to or better than previous studies (Williams 1998). However, to have full confidence in the results discussed here it is necessary to scrutinize in detail a number of issues not touched upon in these tests, but which are key to the evolution of this simulation.
On length scales below the gravitational softening length $\epsilon$ and the SPH kernel radius $h$, the physics of gravity and hydrodynamics respectively are not resolved. In this simulation all gravitational features discussed are much larger than $\epsilon=175$ pc: proto-galactic clumps (one to several kpc), the stellar bulge ( $3 \mathrm{kpc}$ ), sub-galaxies ( 1 to $3 \mathrm{kpc}$ ), disks (20 kpc in radius, although the vertical structure is unresolved), warps (10 kpc), tidal bridges (a few to several kpc), and spiral arms (20 kpc in length, $1 \mathrm{kpc}$ in thickness). One feature on a scale less than $\epsilon$ which has been resolved to the limit of $h$ is the shock front on the inner edge of the spiral arms. However this a hydrodynamic discontinuity associated with the transonic flows induced by the non-axisymmetric potential, and in which gravity does not play a direct role. The shock discontinuity occurs over a scale less than $100 \mathrm{pc}$, comparable to the value of $h$ in the postshock region.

Because $h$ was allowed to evolve freely such that between 30 and 50 neighbours were contained within $2 h$, a small number of particles at the centres of the sub-galaxies (less than 3\%) had very small values of $h$. Typical values within the central $200 \mathrm{pc}$ of the highest density sub-galaxies were of order $60 \mathrm{pc}$, while the smallest values encountered were roughly 5 pc. On these sub- $\epsilon$ scales gravity is not modeled accurately, and furthermore, since such scales are of similar order to the sizes of cold clouds in the ISM, a continuum approximation begins to be inappropriate. Clearly in these regions the physics of self-gravitational hydrodynamics is not modeled accurately, but all the structures relevant to the comparison of these results with observations are of much larger scale, and are accurately modeled. To support this assertion a number of re-runs of this simulation were carried out. In these it was arranged that a typical value of $h$ would not be smaller than roughly $175 \mathrm{pc}$ by decreasing the total number of particles from 130000 , to 32000 in the first re-run, and to 16000 in the second re-run. These simulations can be viewed at http: // www.astro.cf.ac.uk/pub/Alistair.Nelson/ indexgf.html, and Fig. 17 shows a comparison of the star formation rates and final surface density profiles. As a result the SPH resolution within the central $200 \mathrm{pc}$ of the sub-galaxies was degraded to typically 150 and $200 \mathrm{pc}$, with a minimum of 30 and $70 \mathrm{pc}$ respectively. While the details of the fragmentation process differ between the simulations, their gross evolution is very similar, and the resultant numerical galaxies were found to have similar properties and structural parameters. Differences in star formation rates are most apparent during the phase in which star formation in the sub-galaxies dominates the global star formation rate. However the conclusions of this paper are not dependent on the internal structure of these sub-galaxies or their detailed star formation rates, which will both be sensitive to the detailed physics of a multiphase ISM and the mechanisms of star formation. In light of the considerable uncertainties with these issues at present, it is sufficient that these sub-galaxies are recognized as sites of early star formation, formed 

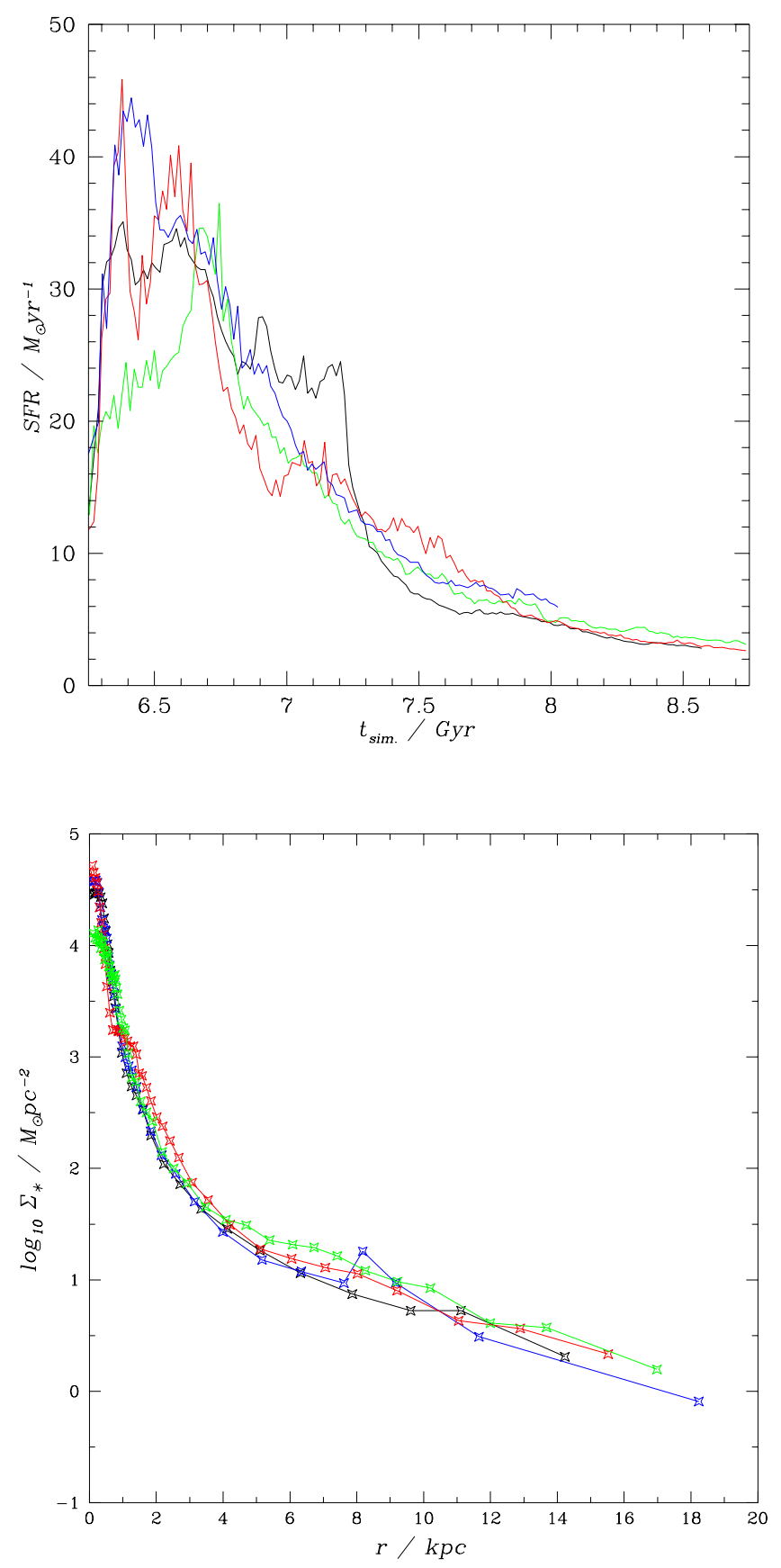

Fig. 17. A comparison of the star formation rate and stellar density profiles in the re-run simulations. The re-runs with: low amplitude Poisson noise (same resolution), no initial noise but with one quarter, and one eighth of the number of particles are shown in red, blue and green respectively. The simulation discussed in detail in this paper is shown in black. Due to the high computational cost incurred, the Poisson-noise rerun had to be terminated at $8 \mathrm{Gyr}$, and thus the stellar surface density profiles shown here were taken at this time. Note that during the epochs in which star formation activity was concentrated within the sub-galaxies, roughly from 6.2 to $6.7 \mathrm{Gyr}$, there is a significant discrepancy in the lowest resolution simulation. However, these differences have little baring on the equilibrium stellar surface density profiles shown. Before the range of timescales shown, the star formation rates were found to be identical. as a consequence of the physics of self-gravitating gas. Thus for the purposes of the analysis discussed in this paper, such limitations have no effect on the conclusions presented here.

Previous authors have commonly constrained $h$ to be greater than $\epsilon$. However there are a number of reasons why this may not be the most appropriate procedure, and may result in unwanted consequences. The particles with $h<\epsilon$ discussed above were also found to have the smallest timesteps in the simulation, and these timesteps were not determined by the $h$ dependent hydrodynamic Courant condition (see Hernquist \& Katz 1989, Eq. (2.37)), but rather the timestep condition determined from accelerations and velocities (see Katz 1991, Eq. (1)). Thus if $h$ were increased to $\epsilon$ for all particles which would have $h<\epsilon$ in our high resolution simulation, keeping the same total number of particles, a large increase in the number of neighbours per SPH summation would result, and thus a large increase in computational cost. In the case of this simulation the required CPU time would have increased from 1.3 to $10 \mathrm{CPU}$ years, while the accuracy and resolution of the hydrodynamical features would have been degraded. The purpose of softening gravity is to allow particles to pass each other on scales less than $\epsilon$ without unrealistic two body effects (Hockney \& Eastwood 1989); while SPH kernel radii $h$ are chosen to ensure that a sufficient number of neighbours are included in summations that stochastic fluctuations are suppressed, and quantities are evaluated to sufficient accuracy (Monaghan 1992). Thus the purpose behind $h$ and $\epsilon$ are distinctly different; and independent of whether or not an $h \geq \epsilon$ condition is implemented, gravity will be inaccurately modeled on scales less than $\epsilon$. On these grounds there would appear to be no strong physical argument for also limiting $h$ to greater than $\epsilon$.

Furthermore, galaxy-forming simulations in which a $h \geq \epsilon$ constraint was imposed have revealed that such a constraint can also affect the large scale characteristics of the resultant numerical galaxies. When excessively large values of $h$ are used in the central regions of a numerical galaxy, SPH summations sample over a large range of velocities. As a result, shear viscosity becomes effective in transporting angular momentum radially outwards and gas mass inwards, and the numerical galaxy becomes unphysically centrally concentrated. It was also found that such a constraint on $h$ results in inaccuracies in all quantities calculated using SPH summations. For example, densities and pressure forces were found to be as much as 100 times smaller when $h$ was constrained. As demonstrated in the simulations discussed here, such problems can be avoided by a) not imposing such a criteria on $h$, and b) using a sufficiently high number of particles.

No initial distribution of noise was used in the simulation discussed here, however it is more appropriate to consider this simulation as having a minimal amplitude of initial noise. The noisy distribution of particles which evolved in this simulation, see Fig. 1, is an artificial artefact of numerical inaccuracies in the 
SPH and Treecode method, propagated in time. Such small random velocities play the same role as a Poisson spectrum of noise with low amplitude. Note that all numerical methods are subject to such truncation errors, although the effects of such errors are usually hidden by the initial non-regular particle distribution. A number of re-runs were carried out to gauge the importance of the spectrum of noise on the conclusions of this paper. The simulation was repeated with each particle displaced from its grid position a small amount in each Cartesian direction, chosen randomly from a Gaussian with zero mean and standard deviation one tenth of the grid separation. The resultant numerical galaxy was found to evolve in a similar fashion to that discussed here, and the equilibrium structural parameters were found to differ by no more than 5\%. See Fig. 17 and the animations http:// www.astro.cf .ac.uk/pub/Alistair.Nelson/ indexgf.html. Thus these re-run simulations show that the conclusions of this paper are not sensitive to the initial distribution of artificial noise, provided it does not have an amplitude as large as that deduced from applying a CDM power spectrum on this scale (see below).

It is not straightforward to compare quantitively this simulation with those published previously, since there are many differences in the implementation of the physics of the ISM and star formation, and the initial configuration of small scale noise in the particle distribution. However, even with these differences, the general evolution and star formation history of this simulation is very similar to those presented by, for example, Katz \& Gunn (1991), Katz (1992), and Steinmetz \& Müller (1994, 1995), which could also have been well described by the five phase paradigm of galaxy formation. There are three main differences in evolution between these simulations and the evolution of that analysed here. Firstly, the simulations of Katz (1992) and Steinmetz \& Müller (1994, 1995) formed significant sub-structure in both the gas and dark matter particle distributions during the expansion and collapse of the proto-galaxy (phase I and II), which resulted in a higher star formation rate than in the simulation analysed here. Secondly, this sub-structure resulted in sub-galaxies with associated dark matter halos, and sub-galaxies which have orbits out of the plane of the forming disk galaxy. However, Katz (1992) reports that such sub-galaxies lose their dark matter halos in the ensuing global collapse. Therefore these evolved sub-galaxies can be considered as essentially the same class of object as the sub-galaxies formed in the simulation analysed here, although their formation mechanism is slightly different. It is notable that the sizes and masses of these sub-galaxies are similar to those found in the minor dwarf satellites of The Local Group (Hodge 1994). Thirdly, this increase in the level of inhomogeneity in comparison to the simulation discussed here results in a more asymmetric potential well. Therefore the galaxy disks are commonly found to form with their angular momentum vectors significantly displaced in direction from the angular momentum vector of their dark matter halo, and have significant warps. In contrast, the low level of in- homogeneity in this simulation produced a galaxy whose dark matter halo and galaxy disk angular momentum vectors were almost aligned: the numerical galaxy disk in this simulation is oriented, to within a few degrees, perpendicular to the initial axes of rotation, although weak warps are observable in the gas disk, see Fig. 5.

Unlike previous simulations, the numerical galaxy discussed here was not found to be deficient in angular momentum, did not have an overabundance of dwarf companions, and did not have a rotation curve inconsistent with observations. All three of these results are due to the minimal level of initial inhomogeneity used. While angular momentum was transferred from the gas to the dark matter component during collapse, see Fig. 10, this effect was not catastrophic. This may indicate that (in addition to ensuring that $h$ is not too large, to avoid artificial angular momentum transfer) decreasing the amplitude of noise on sub-galactic, or even possibly sub-cluster, scales (in comparison to the accepted amplitudes inferred for CDM power-spectrum) is necessary to avoid a high degree of fragmentation and transfer of angular momentum early in a proto-galactic collapse. As was suggested in the introduction, the origins of such a modification to the CDM power spectrum may lie in the importance of the dissipational physics of the pre-galactic gas on these scales; physical processes missing from the CDM power spectrum. Galaxies are intrinsically dissipational objects: it is natural to suppose that the initial conditions from which they formed also had a strongly dissipational element to their makeup.

A number of studies have focused on the importance of the detailed chain of thermodynamic processes at play in a multiphase ISM (Yepes et al. 1997; Hultman \& Pharasyn 1999), and the role of energy feedback from stars (Navarro \& White 1993; Springel 2000). The isothermal model used here does not try to explicitly model these processes, but implicitly assumes that when averaged over length scales of order $h$ and larger, dissipative losses in the ISM are balanced by energy input from stars and supernovae and compressional heating. Thus this model is most appropriately considered as a zeroth order approximation to these more sophisticated prescriptions. While undoubtedly the detailed balance of energy exchange in the ISM does play a role in determining galaxy structure, length scales associated with such processes are of the order of $1 \mathrm{kpc}$ and less, and the gross structural components of a galaxy have characteristic length scales of a few to $20 \mathrm{kpc}$. Thus it is tenable that the formation of these components is well modeled by the isothermal assumption, and the results of this paper confirm this.

Finally, the mechanism of bulge formation suggested by the evolution and formation of the stellar bulge component in this simulation provides a natural, robust manner in which such components may form. The oscillation of cells of gas about the plane of the forming galaxy disk may be expected to occur to some extent irrespective of the specific details of the ISM at that time. Theories forwarded to date on the origin of the bulge component in 
spiral galaxies fall into three broad classes: hypotheses which question the differences between bulge components and other galaxian features such as spiral arms and bars (Kormendy 1993; Wyse et al. 1997); bulge formation by the merger of stellar and gaseous material with an existing galaxy disk (Tremaine et al. 1975; Kauffmann 1996); and bulge growth by secular evolution of a galaxy disk (Hasan \& Norman 1990; Courteau et al. 1996; Noguchi 2000). In contrast to these bulge formation scenarios, the mechanism proposed here is an intrinsic part of the process of forming a galaxy, and is a natural result of the core gravitational and dissipational physics. During the merger of the sub-galaxies (phase IV), it was found that the bulge did grow in a secular fashion similar to that suggested by Noguchi (2000). However this was not the principal mechanism by which the bulge component formed. At the end of the simulation, only $25 \%$ of the stellar mass within $3 \mathrm{kpc}$ of the centre of the numerical galaxy had actually fallen in from outside of this radius; the rest had formed in situ.

Acknowledgements. The authors would like to thank Profs. C. Frenk, P. Hut, and M. Noguchi for supporting the publication of this paper, and Prof. C. Frenk for helpful suggestions on its revision. PRW thanks the Particle Physics and Astronomy Research Council of the UK for the award of a studentship, and The Royal Society and the Japanese Society for the Promotion of Science for the award of a Research Fellowship.

\section{References}

Abraham, R. G., et al. 1994, ApJ, 432, 75

Barnes, J. E. 1994, in The Formation and Evolution of Galaxies, ed. C. Mũnoz-Tuñón, \& F. Sánchez, 399

Barnes, J., \& Hut, P. 1986, Nature, 324, 446

Bertschinger, E. 1985, ApJS, 58, 39

Binney, J. J., \& Merrifield, M. 1998, Galactic Astronomy (Princeton University Press)

Binney, J. J., \& Tremaine, S. 1987, Galactic Dynamics (Princeton University Press)

Buat, V. 1992, A\&A, 264, 444

Bullock, J. S., Kravtsov, A. V., \& Weinberg, D. H. 2000, ApJ, 539,517

Bunker, A. J., \& van Breugel, W. J. M. 1999, The Hy-Redshift Universe, ASP Conf. Ser., 193

Burkert, A. 1995, ApJ, 447, L25

Burkert, A. 2000, ApJ, 534, L143

Casertano, S., \& van Gorkom, J. H. 1991, AJ, 101, 1231

Charlot, S., Worthey, G., \& Bressan, A. 1996, ApJ, 457, 625

Clements, D. L., \& Couch, W. J. 1996, MNRAS, 280, L43

Courteau, S., de Jong, R. S., \& Broeils, A. H. 1996, ApJ, 457, L73

Cowie, L. L. 1980, ApJ, 236, 868

de Bernardis, P., et al. 2000, Nature, 404, 955

de Blok, W. J. G., \& McGaugh S. S. 1997, MNRAS, 290, 533

de Jong, R. S. 1996a, A\&AS, 118, 557

de Jong, R. S. 1996b, A\&A, 313, 45

de Jong, R. S., \& van der Kruit, P. C. 1994, A\&AS, 106, 451

Eggen, O. J., Lynden-Bell, D., \& Sandage, A. R. 1962, ApJ, 136,748

Elmegreen, B. G. 1994, ApJ, 425, L73
Englmaier, P., \& Gerhard, O. 1997, MNRAS, 287, 57

Fall, S. M., \& Efstathiou, G. 1980, MNRAS, 193, 189

Firmani, C., et al. 2000, MNRAS, 315, L29

Flores, R., \& Primack, J. R. 1994, ApJ, 427, L1

Gelato, S., \& Sommer-Larsen, J. 1999, MNRAS, 303, 321

Gingold, R. A, \& Monaghan, J. J. 1977, MNRAS, 181, 375

Gingold, R. A., \& Monaghan, J. J. 1983, MNRAS, 204, 715

Gott, J. R., III, \& Thuan, T. X. 1976, ApJ, 204, 649

Gunn, J. E. 1982, in Astrophysical Cosmology, ed. H. A. Brück, G. V. Coyne, \& M. S. Longair (Vatican: Specula Vaticana), 233

Hannestad, S., \& Scherrer, R. J. 2000, Phys. Rev. D, 62, 043522

Hasan, H., \& Norman, C. 1990, ApJ, 361, 69

Hernandez, X., \& Gilmore, G. 1998, MNRAS, 294, 595

Hernquist, L. 1987, ApJS, 64, 715

Hernquist, L., \& Katz, N. 1989, ApJS, 70, 419

Hockney, R. W., \& Eastwood, J. W. 1989, Computer Simulation Using Particles, Adam Hilger

Hodge, P. 1994, in The Formation and Evolution of Galaxies, ed. C. Mũnoz-Tuñón, \& F. Sánchez, 5

Hoyle, F. 1953, ApJ, 118, 513

Hultman, J., \& Pharasyn, A. 1999, A\&A, 347, 769

Jenkins, A. J., et al. 1998, ApJ, 499, 20

Kaminokowski, M., \& Liddle, A. R. 2000, Phys. Rev. Lett., 84, 4525

Katz, N. 1991, ApJ, 368, 325

Katz, N. 1992, ApJ, 391, 502

Katz, N., \& Gunn, J. E. 1991, ApJ, 377, 365

Kauffmann, G. 1996, MNRAS, 281, 487

Kennicutt, R. C., Jr. 1989, ApJ, 344, 685

Kennicutt, R. C., Jr. 1997, ApJ, 498, 541

Klypin, A. A., et al. 1999, ApJ, 522, 82

Kormendy, J. 1993, in IAU 153, Galactic Bulges, 209

Lake G., \& Carlberg, R. G. 1988, AJ, 96, 1581

Lake G., \& Carlberg, R. G. 1988, AJ, 96, 1587

Larson, R. B. 1969, MNRAS, 145, 405

Larson, R. B. 1974, MNRAS, 166, 585

Larson, R. B. 1975, MNRAS, 173, 671

Larson, R. B. 1976, MNRAS, 176, 31

Larson, R. B. 1988, in Galactic and Extragalactic Star Formation, ed. R. E. Pudritz, \& M. Fich, 435

Larson, R. B. 1992, in Star Formation in Stellar Systems, ed. G. Tenorio-Tagle, M. Prieto, \& F. Sánchez, 125

Lucy, L. B. 1977, AJ, 82, 1013

Lynden-Bell, D. 1967, MNRAS, 136, 101

McKee, C. F., \& Ostriker, J. P. 1977, ApJ, 218, 148

Mestel, L. 1963, MNRAS, 126, 553

Mihos, J. C., \& Hernquist, L. 1994, ApJ, 437, 611

Mobasher, B., et al. 1996, MNRAS, 282, L7

Monaghan, J. J. 1992, ARA\&A, 30, 543

Moore, B., et al. 1999a, ApJ, 524, L19

Moore, B., et al. 1999b, MNRAS, 310, 1147

Navarro, J. F., \& Benz, W. 1991, ApJ, 380, 320

Navarro, J. F., Eke, V. R., \& Frenk, C. S. 1996, MNRAS, 283, L72

Navarro, J. F., Frenk, C. S., \& White, S. D. M. 1995, MNRAS, 275,56

Navarro, J. F., \& Steinmetz, M. 1997, ApJ, 478, 13

Navarro, J. F., \& White, S. D. M. 1993, MNRAS, 265, 271

Navarro, J. F., \& White, S. D. M. 1994, MNRAS, 267, 401

Noguchi, M. 2000, MNRAS, 312, 194

Peebles, P. J. E. 1969, ApJ, 155, 393

Persic, M., \& Salucci, P. 1995, ApJS, 99, 501

Rees, M. J., \& Ostriker, J. P. 1977, MNRAS, 179, 541 
Riotto, A., \& Tkachev, I. 2000, Phys. Lett. B, 484, 177

Roberts, M. S., \& Haynes, M. P. 1994, ARA\&A, 32, 115

Salmon, J. K., \& Warren, M. S. 1994, J. Comp. Phys., 111, 136

Schmidt, M. 1959, ApJ, 129, 243

Simien, F., \& de Vaucouleurs, G. 1986, ApJ, 302, 564

Sleath, J. P., \& Alexander, P. 1995, MNRAS, 275, 507

Sommer-Larsen, J., Gelato, S., \& Vedel, H. 1999, ApJ, 519, 501

Spergel, D. N., \& Steinhardt, P. J. 2000, Phys. Rev. Lett., 84, 3760

Spitzer, L., Jr. 1978, Physical Processes in the Interstellar Medium (New York: Wiley)

Springel, V. 2000, MNRAS, 312, 859

Stark, A. A., \& Brand, J. 1989, ApJ, 339, 763

Steinmetz, M., \& Müller, E. 1994, MNRAS, 281, L97

Steinmetz, M., \& Müller, E. 1995, MNRAS, 276, 549

Theis, Ch., \& Hensler, G. 1993, A\&A, 280, 85
Tremaine, S., Ostriker, J. P., \& Spitzer, L., Jr. 1975, ApJ, 196, 407

van Albada, T. S. 1982, MNRAS, 201, 939

van den Bergh, S., et al. 1996, AJ, 112, 359

von Weizsäcker, C. F. 1951, ApJ, 114, 165

Weil, M. L., Eke, V. R., \& Efstathiou, G. 1998, MNRAS, 300, 773

White, M., \& Croft, R. A. C. 2000, ApJ, 539, 497

Williams, P. R. 1998, Ph.D. Thesis, Cardiff University

Williams, R. E., et al. 1996, AJ, 112, 1335

Wyse, R. F. G. 1986, ApJ, 311, L41

Wyse, R. F. G., Gilmore, G., \& Franx, M. 1997, ARA\&A, 35, 637

Wyse, R. F. G., \& Silk, J. 1989, ApJ, 339, 700

Yepes, G., et al. 1997, MNRAS, 284, 235

Yoshida, N., et al. 2000, ApJ, 535, L103

Young, J. S., \& Scoville, N. Z. 1991, ARA\&A, 29, 581 